\title{
Du chiffonnier à l'anthropologue : statut du texte et positionnement du chercheur sur un terrain littéraire et théâtral
}

\section{Maëline Le Lay}

\section{(2) OpenEdition}

1 Journals

Édition électronique

URL : https://journals.openedition.org/coma/4288

DOI : $10.4000 /$ coma.4288

ISSN : 2275-1742

Éditeur

Institut des textes \& manuscrits modernes (ITEM)

Référence électronique

Maëline Le Lay, « Du chiffonnier à l'anthropologue : statut du texte et positionnement du chercheur sur un terrain littéraire et théâtral », Continents manuscrits [En ligne], 13 | 2019, mis en ligne le 15 novembre 2019, consulté le 12 janvier 2023. URL : http://journals.openedition.org/coma/4288 ; DOI : https:// doi.org/10.4000/coma.4288

Ce document a été généré automatiquement le 12 janvier 2023.

Creative Commons - Attribution - Pas d'Utilisation Commerciale - Pas de Modification 4.0 International - CC BY-NC-ND 4.0

https://creativecommons.org/licenses/by-nc-nd/4.0/ 


\title{
Du chiffonnier à l'anthropologue : statut du texte et positionnement du chercheur sur un terrain littéraire et théâtral
}

\author{
Maëline Le Lay
}

\section{Introduction 1}

1 Dans un de ses derniers textes, « Vertus de l'indiscipline : langues, textes, traductions », Alain Ricard, prolongeant une des nombreuses conversations intellectuelles qu'il avait avec ses amis et collègues, disait de lui-même qu'il était un chiffonnier². Dans ce texte, il délivre sa conception de l'africanisme, de la littérature africaine et il expose surtout la méthode qu'il est le premier - et sans doute un des seuls en France - à avoir défendue dans le champ académique des études littéraires : le terrain ${ }^{3}$ :

Il [le chercheur] est en situation d'apprenant, en situation de faiblesse [...] En somme, le monde nous saute à la figure, à nous de nous en débrouiller: l'indiscipline est une nécessité, nous devons bricoler dans les marges, parfois, et il est vain de vouloir faire entrer à toute force ce que nous observons, ce que nous entendons, dans des cadres préétablis. Or, peut-être est-ce avec la dissonance que commence la connaissance...

Dans ce texte, Alain Ricard prône indistinctement les vertus de l'ethnographie et de l'anthropologie culturelle comme modes d'appréhension de nos objets littéraires et comme modes de connaissance de ces derniers. Or, quoique ces deux disciplines soient toutes deux liées au terrain, elles ne poursuivent pas les mêmes objectifs, ainsi que le rappelle Tim Ingold. Dans Faire anthropologie, archéologie, art et architecture, l'anthropologue écossais s'applique à distinguer les deux pour mieux mettre en valeur les apports - à ses yeux supérieurs - de l'anthropologie ${ }^{4}$. Il ne s'agit pas pour moi de prendre position sur cette question clivante pour les disciplines de terrain mais de 
m'inspirer de cette différenciation épistémologique pour voir comment elle peut nourrir l'épistémologie d'une recherche littéraire menée sur le terrain :

Ce que nous appelons « recherche » ou même «travail de terrain » ressemble, en fait, à une sorte de master class prolongée où les novices apprennent progressivement à voir les choses, à les entendre et à les ressentir [...]. Il arrive toutefois que plutôt que de faire l'épreuve de ce genre d'éducation, certains chercheurs de terrain travaillent à réunir une documentation sur les modes de vie et d'histoire de leurs communautés d'accueil. Ce travail [...] est connu sous le nom d'ethnographie ${ }^{5}$.

Plus loin, il précise :

L'anthropologie consiste à étudier avec et à apprendre de; elle ouvre un processus de vie qui engage une transformation du processus lui-même. L'ethnographie est une étude de et un apprentissage sur, dont les résultats obtenus sur le long terme sont le fruit d'un compte-rendu sélectif répondant à une finalité documentaire ${ }^{6}$.

Cette distinction éclaire singulièrement le travail du/de la chercheur.e en théâtre et en littérature qui se rend sur un terrain donné en vue de percevoir, analyser, comprendre les dynamiques sociales, humaines et littéraires à l'œuvre localement sur une période donnée. En premier lieu, il me semble que le terrain théâtral et littéraire ne peut faire l'économie d'une collecte de matériaux, tâche du chiffonnier si elle en est, souvent entreprise aux premiers temps du terrain. Néanmoins, cette tâche ne se suffit pas à elle-même. Dans la perspective d'une analyse fine des textes dans leur contexte sociopolitique, sociolinguistique et des jeux d'acteurs dans le milieu artistique étudié, le/la chercheur.e ne saurait se contenter de collecter des textes variés trouvés sur son terrain; lesquels, en soi, sont certes déjà porteurs de savoirs sur le terrain qu'il appartient au/à la chercheur.e d'intégrer et reconstituer, mais qui ne suffiront ni à leur rendre toute leur charge signifiante dans ledit contexte, ni à engager un véritable dialogue avec le terrain choisi. Le travail de terrain du/de la chercheur.e en littérature ou théâtre excède en quelque sorte cette étape, il lui faut aller au-delà. Outre cette distinction entre ces deux disciplines de terrain que sont l'ethnographie et l'anthropologie, Tim Ingold apporte une notion fondamentale qui permet de mieux appréhender la densité du travail anthropologique conçu d'abord comme une expérience, comme une étape d'intégration de la connaissance se situant au-delà, voire par-delà le mode de connaissance propre à l'ethnographie répondant exactement aux modèles épistémologiques bien connus auxquels nous avons été formé.e.s : rassembler des informations, les sélectionner, les assembler entre elles selon un système de variables ou de tendances, les traiter selon un mode déductif, les synthétiser pour en présenter une lecture logique, adossée à un ensemble de références, thèses et exemples... Cette notion, centrale dans la pensée d'Ingold, est celle de transformation. La «transformation du processus " se donne à voir à travers la transformation de l'objet initialement ciblé et cerné, cadré selon des bornes théoriques et des représentations du chercheur qui suit une intuition, une piste à partir de traces collectées, qu'il s'agisse d'archives ou de "tin-truck texts ", selon l'expression de Karin Barber ${ }^{7}$, soit des textes amassés dans des cantines de fer blanc, comme autant de fragments textuels conservés par devers soi, jugés par leurs auteurs (ou leurs lecteurs) inadéquats à la publication ou à la performance, bref à la médiation vers un public audience ou lectorat. Cette transformation de l'objet est induite par la transformation du « processus de vie » qui est le but poursuivi par l'approche anthropologique. C'est en engageant son corps et son être entier dans son terrain, dans sa recherche au milieu de ceux et celles qu'il étudie, que l'anthropologue va peu à peu se trouver transformé par 
son expérience et sa relation avec les autres et leur milieu, par la façon dont il va interagir avec cet ensemble dynamique. Car le projet d'Ingold est d'envisager la relation du chercheur avec le monde par le prisme de la notion de correspondance :

Ainsi, l'art de l'enquête avance et se transforme en temps réel, en se mettant au diapason de la vie de celles et ceux avec lesquels l'enquêteur est en contact, et plus largement $\mathrm{du}$ monde auquel tous appartiennent. [...] Mettre en œuvre cette méthode, ce n'est pas décrire le monde ou le représenter mais apprendre à voir ce qui se passe autour de nous de sorte à pouvoir, en retour, lui répondre. Autrement dit, c'est établir une relation avec le monde que j'appellerai dorénavant de correspondance $e^{8}$.

L'analyse de ces correspondances et interactions, de l'écart entre ses propres attentes initiales et ce qui se passe effectivement dans les faits, doublée de l'analyse des attentes de ses interlocuteurs, va nécessairement impacter l'objet de sa recherche, impliquant une reconfiguration de son objet et de son mode d'acquisition, d'intégration puis de transmission de la connaissance et la compréhension acquises. Autrement dit, l'anthropologue collectant textes et récits sur le terrain peut difficilement faire l'économie d'une démarche réflexive dans le traitement de son sujet d'étude. Quoique cette démarche ne soit pas nouvelle en anthropologie - elle a été bien théorisée dans les années 1980 par les tenants du «cultural turn» tels que Clifford Geertz ou James Clifford $^{9}$ (pour ne citer que deux des anthropologues les plus versés dans une réflexion sur l'écriture anthropologique et les liens qu'elle entretient avec la littérature), puis, plus récemment du "ethical turn $»^{10}$ - elle est davantage popularisée dans les études anglo-saxonnes. Dans le domaine francophone, elle jouit aujourd'hui d'un certain regain en sciences humaines (anthropologie, études littéraires et théâtrales, histoire, géographie...), comme en témoigne par exemple le travail d'Aurore Vermylen, PierreJoseph Laurent, Didier Nativel, ou Jean-Baptiste Lanne ${ }^{11}$ (voir infra).

6 En définitive, le chercheur qui se rend sur le terrain a nécessairement l'ambition de trouver des textes dont il ne peut, depuis le lieu d'où il formule ses hypothèses d'après une intuition personnelle, soupçonner l'existence. Du moins, des textes dont il ne peut deviner la forme, encore moins leurs mises en scène (quelles langues? Quelles conventions théâtrales? Quels publics?...). Le chercheur qui s'engage sur un terrain théâtral et littéraire a en quelque sorte l'ambition de trouver ce qu'il ne connait absolument pas, sans être sûr du tout de trouver quoi que ce soit de différent de ce dont il a déjà pris connaissance à distance grâce à la documentation consultée en amont ${ }^{12}$. En bref, il est à la recherche de ce qui est inaccessible dans les bibliothèques universitaires qui constituent le vivier premier de tout chercheur et une première base qui va lui servir à brosser un état des lieux sommaire et nécessairement imparfait et incomplet du paysage littéraire et théâtral du terrain choisi. Un vivier que, dans le cas de la catégorie que l'on appelle "théâtre africain", il va bien vite épuiser après avoir compulsé les sources principales du théâtre composé en Afrique francophone ou des traductions, essentiellement de l'anglais : la série de pièces de "classiques » africains ${ }^{13}$ publiées chez Hatier, Monde Noir, Pierre-Jean Oswald (Ngugi wa Thiong'o, Wole Soyinka, Bernard Dadié, Cheikh Aliou Ndao, pour n'en citer que quelques-uns) ou, de manière isolée, dans quelques autres maisons d'édition non spécialisées Afrique (Koffi Kwahulé aux Éditions théâtrales, Dieudonné Niangouna aux Solitaires Intempestifs), ou bien encore dans des maisons non spécialisées en théâtre (Mamadou Mahmoud N'Dongo chez La Cheminante, Sinzo Aanza aux éditions Nzoi). Ajoutons à ce tour d'horizon les " pièces de festivals », c'est-à-dire des pièces publiées à l'occasion de leur 
mise en spectacle lors de festivals dans le cadre de programmation d'un théâtre (voir par exemple la collaboration entre le Tarmac et Lansman ou entre Le festival des Francophonies en Limousin et Lansman); enfin des pièces publiées en Afrique par des éditeurs locaux, par conséquent essentiellement disponibles localement mais jouissant néanmoins d'une certaine visibilité sur la toile (par exemple les éditions Nzoi à Paris/ Kinshasa et les éditions Découvertes du Burkina, collection Récréâtrales).

Une fois donc épuisé ce vivier de textes conservés dans les bibliothèques de proximité, le chercheur qui ne s'en contentera pas s'en ira chercher au-delà de ces lieux de dépôt les plus accessibles, repérer et identifier d'autres textes qui émaneraient plus directement de son terrain. Celles et ceux qui s'engagent dans cette quête le font de différentes manières, passant par la co-composition de textes théâtraux, leur édition (critique), le travail d'archives ou bien encore la caméra. Alain Ricard, par exemple, revêtait volontiers plusieurs casquettes, collectant des textes écrits localement dont certains épuisés et écrits en différentes langues (selon sa vocation première et revendiquée de chiffonnier), transcrivant et éditant des textes de théâtre en ewe semioraux (pièces de concert-party du Togo) ${ }^{14}$ ou encore filmant les spectacles ${ }^{15}$. Johannes Fabian et Karin Barber incarneraient davantage la figure du passeur, transcrivant et traduisant en anglais des textes dont la seule trace écrite est généralement un canevas sommaire. Des textes performés en langues locales (respectivement swahili et yoruba), flanqués d'un appareil critique très fouillé et auxquels s'adosse tout leur savoir anthropologique acquis en vivant avec les gens composant les sociétés étudiées, les habitants de la Copperbelt katangaise pour Fabian et les Yorubas pour Barber ${ }^{16}$. Enfin, l'historien du théâtre ira à la rencontre d'un gisement de textes et de manuscrits plus ou moins griffonnés et opérera une coupe transversale dans les archives théâtrales, comme l'a fait Céline Gahungu avec les archives du concours inter-théâtral de $\mathrm{RFI}^{17}$, ou encore Tobias Warner et Brian Valente-Quinn avec les archives de l'école normale William Ponty au Sénégal, forge de nombreux écrits à vocation littéraire et théâtrale ${ }^{18}$.

Il s'agit pour moi dans cet article de m'interroger d'une manière réflexive sur les méthodes employées et adoptées - parfois intuitivement - pour la compréhension et l'analyse de mes terrains d'étude: du sud-Katanga au sud-est de la République démocratique du Congo où j'ai mené ma recherche doctorale (principalement à Lubumbashi, deuxième ville du pays) à la région des Grands Lacs africains où je travaille actuellement. Cette recherche porte sur l'activité littéraire et théâtrale dans les principales métropoles situées de part et d'autre des lacs Kivu et Tanganyika, en RD Congo (Goma, chef-lieu du Nord-Kivu et Bukavu, chef-lieu du Sud-Kivu), au Rwanda (Kigali) et au Burundi (Bujumbura). D'une recherche l'autre, le théâtre est une pratique centrale dans mon analyse du paysage littéraire de la région étudiée, même si je la saisis dans un mouvement miroir tel un chiasme. En effet, au Katanga, j'ai rétréci mon prisme d'analyse de la littérature sous toutes ses formes (qui était le projet initial) au théâtre, tandis que dans les Grands Lacs, je l'ai élargi au contraire, partant du théâtre pour l'étendre à la littérature sous toutes ses formes (fiction narrative, poésie, slam, théâtre). Pour autant, trois aspects sont communs aux deux recherches : l'attention à la situation sociolinguistique propre aux lieux étudiés, l'attention à l'inscription géographique et sociale de l'activité littéraire et théâtrale dans un paysage donné ainsi que l'analyse de la façon dont la littérature fait société, c'est-à-dire la manière dont elle constitue une activité sociale dans les deux sens du terme. Une activité sociale au sens de sociabilité parce qu'elle est structurée par une organisation sociale impulsée par une dynamique collective de faire-ensemble, de se réunir pour créer et rendre visible 
l'activité littéraire et théâtrale ; une activité à vocation sociale parce qu'elle se donne pour ambition première de participer à l'avancement de la société vers un état meilleur et harmonieux, qu'il se donne "paix ", "réconciliation ", « réparation » ou « cohésion sociale ». Cette optique implique une commune approche des textes dont la première étape est l'observation fine de ce qui se dit et s'écrit dans le lieu dans un large spectre de genres; il s'agit d'un repérage et d'une analyse des formes et usages d'une parole « littéraire »/ théâtrale ou performative. Inévitablement émergent alors les questions du statut et de la nature du texte, de l'absence ou parfois de la difficulté d'accès (pour de multiples raisons) aux archives, manuscrits et autres supports textuels qui constituent ordinairement le matériau premier de la chercheuse en littérature et, dans une moindre mesure, en théâtre. Par conséquent, outre les apports des études théâtrales et littéraires (Angenot, Macé, Grésillon), sont ici mobilisées des notions et méthodes issues de deux courants de l'anthropologie: l'anthropologie culturelle, et plus précisément l'anthropologie textuelle (Fabian, Barber et Ricard), ainsi que l'anthropologie du sensible (Laplantine et Ingold), à l'aide d'exemples tirés des recherches de collègues dans lesquelles je vois des similitudes avec les miennes.

Avant d'examiner le contenu de la besace du chiffonnier du théâtre en m'intéressant notamment à son mode de collecte des textes, je procéderai à une mise au point théorique nécessaire sur la notion de texte (de théâtre), sur la méthodologie et l'approche qu'implique la conception du texte que je défends, en discutant notamment des apports de l'anthropologie et de la génétique textuelle. Puis, après avoir mis à jour les problèmes épistémologiques et éthiques posés par l'étude d'un corpus non canonique composé de textes que l'on participe à créer - notamment en les "fixant " sous une forme théâtrale orthodoxe - j'esquisserai, dans le troisième temps de cet article, des pistes de modes de pensée et de faire avec ces textes - archives, traces écrites, fragments de discours (semi)oraux - comme autant de propositions de maniement de ces textes « rencontrés » (trouvés, collectés, en partie composés) lors de terrains littéraires et théâtraux en m'appuyant sur ma propre expérience en Afrique des Grands Lacs (RD Congo, Rwanda, Burundi).

\section{Dans la besace du chiffonnier du théâtre. Le chiffonnier comme créateur ou qu'est-ce qui fait texte de théâtre?}

"Comment trouve-t-on un texte? ", me demande-t-on souvent lorsqu'on m'interroge sur ma pratique. L'interlocuteur lambda qui cherche à savoir en quoi consiste mon travail d'un point de vue pratique prend appui sur le préjugé - largement servi par la maxime populaire "africaine » attribuée à Amadou Hampâté Bâ : «En Afrique, quand un vieillard meurt, c'est une bibliothèque qui brûle ». Cette formule ressassée à l'envi par l'amateur désireux de caractériser la "culture africaine » revient à dire qu'il n'existe pas de livres et donc de textes en Afrique ${ }^{19}$. Suivant la même logique, s'il n'y a pas de livres ni de textes écrits et imprimés, il ne doit pas non plus y avoir de théâtre(s), laquelle discipline, dans la conception européenne, s'articule encore beaucoup autour du texte (même si les performances et autres spectacles moins texto-centrés se généralisent de plus en plus sur les scènes européennes). De fait, les espaces urbains d'Afrique des Grands Lacs où je travaille sont moins pourvus en librairies et bibliothèques que les espaces européens par exemple ; quant aux plateaux de théâtre, 
ils ne sont pas immédiatement visibles et accessibles puisqu'ils se situent rarement dans des salles conçues pour le théâtre, en nombre limité dans ces villes. Difficile de prétendre le contraire même si la conception dominante - des étrangers, comme des habitants de ces espaces urbains, dans les Grands Lacs tout du moins - tend à exagérer la pénurie de livres et la difficulté d'accès à ce bien culturel d'un côté, la présumée absence de théâtre de l'autre, sous prétexte que les textes imprimés dramatiques et les salles dédiées au théâtre ne sont pas légion. À Goma par exemple, lors des assises du théâtre au Kivu co-organisées en 2015 par Yole !Africa et l'ONG Search for Common Ground, la plupart des participants se lamentaient de l'absence de lieu consacré et avançaient cette raison pour expliquer « l'extinction » du théâtre à Goma (ainsi que le voulait le diagnostic posé collectivement) ${ }^{20}$. Il semble qu'il soit difficile, pour plusieurs d'entre eux, de concevoir le théâtre en dehors d'une salle fermée et bien isolée, dotée d'une vraie scène (et non d'un podium de fortune) et pourvue de l'équipement scénique de base (lumière et son, coulisses). La récente mise en scène à Goma en juin 2019 par Patrick Zézé du Soliloque du Roi Léopold de Mark Twain en témoigne une nouvelle fois. L'équipe (mais surtout le metteur en scène) se montrait quelque peu rétive à l'idée de jouer en plein air pour exploiter le potentiel architectural de l'Institut français qui fournissait pourtant un décor adéquat à la pièce.

\section{Faire texte}

En fait, ce malentendu général et relativement bien partagé est le fruit de l'acquisition d'un savoir situé sur la littérature, d'une conception culturellement marquée de la « res literae », comme l'a parfaitement montré Karin Barber, anthropologue du texte et du théâtre, spécialiste du théâtre populaire yoruba. Dans son lumineux essai, The Anthropology of Texts, Persons and Publics dans lequel elle pose d'emblée comme postulat inaugural que la textualité ne se réduit pas à l'écriture ${ }^{21}$, les textes locaux, oraux comme écrits, en langues européennes comme en langues africaines, étant envisagés comme des processus complexes d'entextualisation, plus que comme des artefacts littéraires. Après avoir souligné les limites de l'histoire littéraire de tradition européenne focalisée sur la canonisation d'œuvres selon des critères de littérarité hérités du classicisme, et pointé les limites de l'exploitation des textes par l'anthropologie (qui se contente souvent, à l'instar des autres sciences humaines inspirées par la littérature, d'y puiser comme en un vivier d'exemples illustratifs de théories des faits sociaux), l'auteure analyse les conditions de passage d'un discours à un texte. Il s'agit d'étudier la manière dont un discours est textualisé pour perdurer dans le temps à travers un ensemble de procédés qui visent à rendre le texte disponible pour la répétition, l'improvisation, la recréation et par là, la transmission, par-delà les époques et les foyers de création :

People coming from a background in European literature may be tempted to assume (and often have done so) that every culture has a category corresponding to 'literature'; that all 'literature' is characterized by unity, fictivity, poetic language, or a particular quality of imagination; and that attending to these qualities is the way to get the point of the text. But one of the aims of the anthropology of texts is to open up the sheer range of relationships they can establish between speaker/ writer and hearer/reader, and the ways in which they can be valued and held to have meaning. A central contention of this book is that if a verbal text is to "tell us" anything about a society, social experience, or cultural values, this can only be through its specific textuality, its specific way of being a text, not by by-passing it. ${ }^{22}$ 
Cette conception de la littérature et du texte est complètement à rebours de celle communément enseignée en Europe et en Afrique, dans des pays anciennement colonisés, lesquels ont, pour la plupart, conservé l'essentiel des curricula issus de la période coloniale. La littérature (un terme auquel Karin Barber préfère celui de textualité) est ici conçue comme un processus ouvert et dynamique, un agencement en réseau de relations branchées aux autres sphères du discours, de la vie sociale et de l'espace public. Cette conception confère à la littérature une dimension éminemment plastique qui se prête bien à l'appréhension des textes glanés sur nos terrains littéraires et théâtraux, textes souvent "hors-normes", hétérodoxes, au statut incertain et à la forme évolutive. Elle nous permet alors d'élargir le spectre de notre regard sélectif et discriminant - des genres et formes tels qu'ils nous apparaissent et que nous les percevons dans un premier temps - et nous conduit ainsi à porter une attention singulière à tout type de parole publique requérant et mobilisant un public, à toute théâtralité ou, pour le dire autrement, à toute performance. Dès lors que l'on admet avec Karin Barber que toute performance n'existe pas seule en soi comme un énoncé éphémère et évaporé sitôt proféré, qu'elle est performance d'un texte ${ }^{23}$, l'attention peut se porter sur les modalités d' «entextualisation » (ou de façons de faire texte, de devenir-texte) pour en identifier, décrire et analyser les formes et les pratiques, les styles dans le sens précis que lui donne Marielle Macé dans Styles. Critique de nos formes de vies: des modes d'être ou modalités ${ }^{24}$.

\section{La répétition, genèse du texte théâtral ?}

13 Ces styles sont donc autant de textes au sens où l'entend Barber, autant d'agencements de fragments de discours, de modes de dire et de faire. Si cette approche s'avère si pertinente pour comprendre les textes que nous extrayons de nos terrains, c'est que la nature même du texte de théâtre s'y prête comme naturellement. En effet, même dans des contextes où le texte théâtral imprimé est inscrit dans les usages courants, validé par une sorte de tradition ou plus simplement d'habitude, où il fait (encore) autorité, il ne constitue pour autant qu'une partie du texte final, c'est-à-dire de la performance. Ce postulat, au fondement des études théâtrales, fait l'objet d'une analyse particulièrement approfondie par les anthropologues du théâtre, telle Gay McAuley. Dans son article, "Not Magic but Work. Rehearsal and the Production of Meaning" (comme dans d'autres de ses textes, du reste), elle s'intéresse à la répétition dont elle souligne l'importance dans le processus de création théâtrale ; la répétition, cet espacetemps singulier générant des traces - parfois écrites, griffonnées - qui participeront à constituer l'ensemble du texte final, la performance : «Vitez understood the way that things explored during rehearsal, even if they are not finally included in the performance, can nevertheless leave traces for the actors, and that these greatly enrich the performance $»^{25}$. S'appuyant sur les intuitions et parfois sur les pratiques des metteurs en scène - ses contemporains dont elle observe le travail durant des répétitions ou ceux d'hier qu'elle ne connaît que via leurs écrits, comme Vitez - elle en vient à revendiquer la pertinence d'emprunter à l'ethnographie son mode d'observation, d'analyse et d'écriture :

Perceiving rehearsal in this way, as a site of complex interpersonal relations, a workplace, a crucible facilitating collective creativity, requires a very different approach from the essentially hermeneutic endeavour described in the preceding paragraph. Traditional theatre scholarship provides little methodological guidance 
for dealing with such a task, but ethnography has been a rich source of analytical concepts, procedures and insights. I have taken the concept of «thick description » from Clifford Geertz, and it is this that most encapsulates what I think the study of rehearsal needs to address. ${ }^{26}$

14 On serait forcément tenté de voir en la répétition non seulement, comme elle le souligne explicitement, un terrain d'observation privilégié où mener une observation participante comme le ferait l'anthropologue, mais également le lieu-même du foyer de la création théâtrale, son nid génétique en quelque sorte, une arène ou un bouillon de culture où observer en direct les arcanes de la conception du texte final. Et de fait, la tournure que prendra finalement la création est le fruit de l'ensemble des exercices pratiqués durant la répétition de théâtre et surtout de leur ressenti, de la manière dont celui-ci a été exprimé par l'équipe (metteur en scène, comédiens, parfois dramaturge, scénographe, costumière, régisseur son et lumière etc.), des propositions de jeu et de mise en scène par ladite équipe et que le metteur en scène choisira de conserver ou pas, de noter et ainsi figer sur le texte de théâtre ou sur son carnet de création. Partant, le chercheur en théâtre qui a la chance d'assister aux répétitions voit se jouer devant ses yeux non seulement le déroulé de la pièce elle-même, de manière plus ou moins linéaire, mais encore l'ensemble des propositions et des interactions correspondantes constituant in fine le texte de théâtre, le spectacle tel qu'il sera présenté au public. Ces données l'informeront considérablement sur les différentes dynamiques à l'œuvre dans la création: dynamiques humaines (affects et jeux de pouvoir) et dynamiques esthétiques (orientations et parti-pris, tâtonnements et balancements entre genres et styles différents...). C'est en tout cas le pari que fait Josette Féral dans l'introduction au dossier «Genetics and Performance» qu'elle a coordonné pour la revue Theatre Research International :

What stages does a text go through before publication? When and how does the author hesitate, make mistakes, take detours and make decisions? Focusing on the writing « in process » and on the creative work behind literature, genetic analysis proposes that the writing process itself be analysed, using whatever traces remain in the form of manuscripts or drafts, for example ${ }^{27}$.

\section{Quels textes dans l'improvisation théâtrale?}

Néanmoins, en dépit de l'analogie tentante, au niveau symbolique, entre l'espace-temps singulier de la répétition de théâtre et la forge du texte, son foyer génétique, il reste que ce sont les traces écrites de la pièce ou du spectacle, les éléments tangibles donc, qui forment la matière première du généticien. Or ces traces "trouvées", collectées par le chercheur, qu'il lui appartiendra ensuite de déchiffrer, d'ordonner et d'interpréter, ne sont pas si courantes dans bien des pratiques théâtrales du continent, une part souvent importante de la création étant accordée à l'improvisation. Les théâtres tantôt qualifiés d' "utiles », de "sensibilisation ", " pour le développement " (de TfD, Theatre for Development), largement pratiqués de part et d'autre du continent se basent tous, à proportions variables, sur la méthode inventée et théorisée par le dramaturge et metteur en scène brésilien, Augusto Boal, le théâtre de l'opprimé, dont la forme la plus répandue est le théâtre-forum ${ }^{28}$. Cette théorie marxiste de la performance à vocation sociale se fonde sur une croyance, ou plutôt un vouloir-croire, en les propriétés performatives du théâtre dont les conditions de possibilité sont d'une part l'improvisation, d'autre part l'implication d'acteurs ordinaires et non professionnels. Quoiqu'importées en Afrique à la fin des années 1970 (leur 
rayonnement se situant plutôt dans la décennie 1980-1990), ces pratiques théâtrales inspirées de la méthode de Boal sont toujours utilisées aujourd'hui, avec une fortune et une vigueur variables d'un pays à l'autre ${ }^{29}$.

L'ASBL ${ }^{30}$ " Théâtre \& Réconciliation », basée à Bruxelles mais déployée tant en Belgique qu'en Afrique des Grands Lacs, est, à sa façon, héritière (ou cousine) de ces méthodes. La metteuse en scène Frédérique Lecomte, sociologue de formation formée au théâtre par Jean Louvet, fondateur du théâtre prolétarien à La Louvière, au cœur de la Wallonie industrielle, dans les années 1960, a monté son asbl en 1994 et a depuis créé de nombreux spectacles au Burundi, et, plus récemment, au Rwanda et à l'Est de la RD Congo. La plupart de ses créations lui ont été commanditées par les ONG œuvrant à la "réconciliation» et la «résolution pacifique des conflits", mots d'ordre inscrits à l'agenda politique de cette région depuis les années 1990 qui ont vu la région se déchirer sous le coup d'une série de guerres entremêlées: la guerre au Rwanda (1990-1994) dont le point d'orgue fut le génocide des Tutsi (1994), la guerre au Burundi (1993-2005) puis les guerres du Congo (1996-1997, 1998-2003) suivies d'une guerre larvée au Sud- et au Nord-Kivu (provinces de l'Est congolais, frontalières du Rwanda et du Burundi) qui se poursuivent jusqu'à aujourd'hui, impliquant plusieurs pays voisins ${ }^{31}$.

La méthode de Frédérique Lecomte employée dans Théâtre \& Réconciliation, exposée dans l'ouvrage collectif éponyme qui en constitue une sorte de manifeste ${ }^{32}$, consiste à créer du texte à partir des improvisations des acteurs, lesquels sont rarement des comédiens professionnels puisque son objectif est d'accompagner les "vulnérables » pour leur permettre de s'ouvrir en identifiant les schémas et modes d'action destructeurs, en exprimant leurs propres peines et frustrations, voire leurs traumas, mais aussi en reconnaissant ceux des autres. Cette méthode a été conçue pour redonner confiance, dignité et espoir aux vulnérables. La catégorie de "vulnérables » est, dans son acception, suffisamment large pour accueillir aussi bien des réfugiés et demandeurs d'asile, des homosexuels, des immigrés de la première ou seconde génération, femmes issues de milieux modestes en Europe occidentale, que des enfants-soldats démobilisés, des filles-mères victimes de violences sexuelles, voire des citoyens ordinaires ayant enduré des conflits intenses et meurtriers en Afrique des Grands Lacs.

Mais la spécificité de la méthode de Frédérique Lecomte est moins l'improvisation en tant que telle que sa manière singulière d'utiliser la matière née de l'improvisation pour créer le texte du spectacle qui sera joué, texte variable d'une représentation à l'autre. Ce n'est pas tant le fait de tisser entre elles les scènes nées de l'improvisation qui est original que le montage en live de celles-ci. Le jour de la représentation, les comédiens ont intégré les différentes scènes nées des improvisations faites en langues locales (kinyarwanda au Rwanda, kirundi au Burundi, swahili au Congo) lors des répétitions, chacune caractérisée et identifiée par un geste auquel est associée une chanson. C'est-à-dire que chaque scène a été travaillée jusqu'à ce que la metteuse en scène en soit satisfaite et que le comédien y soit à l'aise mais le montage des scènes entre elles n'est jamais le même, la metteuse en scène improvisant en direct l'enchaînement des scènes. Les comédiens ne connaissent à l'avance que la scène d'ouverture et la scène de clôture. Pour le reste du spectacle, ils devront se laisser guider par la metteuse en scène, debout à l'autre extrémité de la scène, leur faisant face et dessinant, par ses gestes, ses injonctions et interpellations, l'ordre des scènes préalablement intégrées comme autant d'entités autonomes. Frédérique Lecomte ellemême ne connaît jamais par avance l'ordre des scènes, c'est lors du spectacle qu'elle 
l'établira en l'improvisant de manière intuitive. Son credo est d'être à l'écoute du rythme du spectacle. Aussi est-ce la perception qu'elle en a qui guide le choix de telle ou telle scène piochée dans son cahier ouvert à ses pieds dans lequel sont listées les scènes indiquées par les premières paroles de la chanson associée à chacune et coloriées par thématiques et intensité dramatique. Sa perception sur le moment du pouls de la représentation, de l'énergie qu'elle dégage est son bâton de cheffe d'orchestre. Car c'est bien d'une forme de super-orchestration et même quasiment de la création en live, qu'il s'agit. En effet, alors que le chef d'orchestre travaille sur de la matière aboutie, sur des parties de la pièce musicale qui s'enchaînent d'une manière immuable, ici la mise en scène n'est jamais complètement finie, figée, aboutie ; tout change sur place et sur le moment. Le script est ici d'autant plus volatile qu'il ne sert que de mince support, comme un fil sur lequel la metteuse en scène enfile et assortit ses perles entre elles dans un ordre et sur un motif chaque fois différents. Le résultat en acte et en jeu est une sorte de puzzle mouvant se recomposant d'une performance à l'autre.

19 Quoique cette méthode soit propre à Frédérique Lecomte, quiconque s'intéresse au théâtre en Afrique rencontre nécessairement à un moment sur son chemin ce type de théâtre qualifié de différentes manières selon les contextes: TfD, théâtre de sensibilisation, théâtre participatif, théâtre utile..., tous étant des théâtres essentiellement basés sur l'improvisation à partir d'une trame en général écrite, en tout cas fixée sur papier dans les grandes lignes, fût-ce par une liste de mots-clés ou une palette de coloriages comme seuls indices de sens et d'ordre du rythme et des thématiques traitées.

On le voit, le texte de théâtre, et a fortiori le texte de théâtre créé à partir d'improvisations, se prête difficilement à une étude génétique, et même à une stylistique, appliquées à des textes plus canoniques, plus écrits et imprimés, composant les corpus des études littéraires. Se pose donc un problème d'adéquation de la méthode d'analyse textuelle (et génétique) à ce type de textes volatiles, mouvants, résolument ouverts, qui ne se laissent guère fixer. À partir de quels critères décide-t-on alors qu'un texte est texte, même au sens minimal et novateur où l'entend Karin Barber, comme un élément figé du discours? Selon elle, l'entextualisation est conçu comme un processus de "givrage » (freezing) de l'énoncé qui le rend alors disponible à son ré-usage dans d'autres contextes :

Text is created when instances of discourse, by being rendered detachable from their immediate context of emission, are made available for repetition or recreation in other contexts. In other words, they are stretches of discourse which can be reproduced and thus transmitted over time and space ${ }^{33}$.

21 Dans cette perspective, je propose de qualifier les chœurs qui rythment les spectacles de Théâtre \& Réconciliation de textes ${ }^{34}$. Pour tous les spectacles qu'elle réalise dans les Grands Lacs, Frédérique Lecomte travaille exclusivement avec un seul musicien, JeanClaude Minani, un Burundais qu'elle fait venir partout où elle crée un nouveau spectacle, que ce soit au Burundi, au Rwanda ou au Congo. Il est donc l'unique compositeur des chansons qu'il commence par entonner seul pour donner le la aux comédiens qui lui emboîtent rapidement le pas, au son de l'ikembe (lamellophone utilisé dans toute la région d'Afrique des Grands Lacs et centrale) dont il est également le seul à jouer. Ces chansons, presque toujours entonnées en chœur, sont notées sur le cahier de la metteuse en scène (du moins, les premiers mots en swahili orthographiés phonétiquement, Frédérique Lecomte ne parlant pas swahili) et fréquemment 
réutilisées d'une création à l'autre, légèrement adaptées au nouveau contexte, l'essentiel de la mélodie et du texte demeurant inchangé. Dès lors, on peut considérer que les chœurs constituent en effet des instances détachables du discours théâtral à la forme mouvante, réutilisées dans d'autres contextes socio-géographiques.

\section{Modalités de considération et d'exploitation des textes : questionnements méthodologiques}

Quels que soient les critères de catégorisation de ces fragments de discours en textes, dès lors qu'ils ne correspondent pas à la définition orthodoxe du texte, se posent des questions méthodologiques qu'il convient d'affronter pour justifier les corpus proposés à l'issue de terrains littéraires et théâtraux.

Les chercheurs qui choisissent d'étudier des pièces de théâtre bâties à partir de l'improvisation ont en général recours à la transcription de la performance, support scriptural qui constituera dès lors la base principale sur laquelle ils s'appuieront pour mener leur analyse et puiser leurs exemples. Ce faisant, ils fixent par l'écrit les termes et atours d'une performance, laquelle, même si elle est dupliquée dans différents contextes, n'est pas vouée à être répétée à l'identique. En la fixant par écrit et en soumettant cette transcription à leur analyse, ils transforment alors l'objet initial - la performance à partir d'un support scripturaire, un canevas souvent sommaire - en la mettant au même niveau qu'un texte préalablement écrit. Cette opération consistant à créer partiellement son corpus (et non simplement à en collecter les éléments et à les assembler) ne va pas sans poser une série de problèmes - éthiques, déontologiques et épistémologiques - qui se sont déjà posés à certains chercheurs tels qu'Alain Ricard ou Johannes Fabian.

\section{Forcer le passage, créer des textes et des corpus}

Alain Ricard est, à ma connaissance, le premier chercheur en littérature du monde académique français à avoir travaillé, pour sa thèse de littérature comparée, sur un corpus mixte fait de textes écrits et publiés et de textes semi-oraux enregistrés, transcrits et traduits par ses soins. Dans cette thèse d'État réalisée au Togo et soutenue à Bordeaux en 1981, il étudia la diglossie littéraire à l'œuvre dans les romans publiés de Félix Couchoro (en français) et dans les spectacles de concert-party (en ewe). Cette démarche s'inscrivait doublement en rupture avec les usages et les conceptions de la littérature en vigueur dans les études littéraires et les curricula afférents, qui n'avaient pas encore été détrônés à l'époque, loin s'en faut. De fait, lorsqu'il menait ses recherches dans le Togo des années 1960-1970, le concert-party était un spectacle dont le public togolais était friand mais qui ne jouissait d'aucune considération chez ses collègues enseignants et étudiants à l'Université de Lomé ${ }^{35}$. Du fait que ces spectacles ne répondaient pas aux normes du théâtre dit « classique » (en français, interprétation d'une pièce de théâtre du répertoire classique français, éventuellement d'Afrique francophone, pièce canonisée par les systèmes littéraires francophones, caractérisée par un certain formalisme en termes de mise en scène, par une conception très normée de l'intrigue et du dispositif théâtral spatio-temporel), le milieu togolais éduqué ne pouvait envisager de l'intégrer dans un corpus littéraire académique. Alain Ricard, passant outre les différences formelles évidentes d'avec le théâtre dit «classique » en 
français tel que pratiqué au Togo sur les scènes officielles, considéra d'emblée le concert-party comme du théâtre, y voyant d'abord et avant tout une fiction mise en scène, des histoires racontées et interprétées par des personnages à forte connivence avec le public, des jeux sur la langue. Il fit alors le choix de les traiter au même niveau d'égalité avec des textes dont la légitimité n'était pas remise en question par le milieu intellectuel local, ceux de Félix Couchoro, écrivain feuilletoniste publiant ses histoires en français dans différents journaux, Togo-Presse notamment ${ }^{36}$. Il s'agissait là d'un partipris fort à la fois eu égard à sa propre institution, le monde académique français, mais aussi, et peut-être même surtout, au regard de l'environnement local où il ancrait son étude, des conceptions de la langue et la littérature qui y dominaient et vis-à-vis desquelles il s'inscrivait ouvertement en porte à faux. Sans doute faut-il déjà voir dans ses premières recherches, le mélange de provocation et de militantisme qui le caractérisaient, lui qui n'eut jamais de cesse de lutter contre une conception hégémonique des langues européennes (et singulièrement du français) dans l'approche des littératures de l'Afrique. Il ne se lassa jamais de fustiger ce biais épistémologique dans lequel il voyait le dernier relent d'un colonialisme suranné et cette révolte, parce qu'elle était politiquement légitime à ses yeux, le dispensait sans doute de prendre pleinement la mesure du point de vue émique des effets du bouleversement épistémologique que son travail déclenchait ainsi dans le milieu académique et intellectuel togolais. ${ }^{37}$

Quand dans les années 1960 et 1970, Alain Ricard renouvelait au Togo, le mode de composition d'un corpus littéraire et théâtral en composant, avec le concours des comédiens et étudiants, des textes ewe après les avoir enregistrés, transcrits et traduits en français, Johannes Fabian menant ses recherches dans la province du Shaba (actuel Katanga) du Zaïre de l'époque, innovait pareillement en proposant de passer d'une " ethnographie informative à une ethnographie performative ». Prenant ses distances à la fois avec l'approche herméneutique et l'approche formaliste témoignant toutes deux selon lui d'un certain « fondamentalisme textuel $»^{38}$, Johannes Fabian propose d'éclairer un point aveugle de chacune de ces approches qu'est ce « vast body of texts on which anthropologists base their writing » qu'il nomma les « textes ethnographiques » :

They consist above all [...] of protocols made of actual communicative (mostly verbal) exchanges, as well of "performances", ranging from storytelling, to poetic and mythical recitations, to rituals, records of transactions, and litigation. Other types can of course be added to this list, such as autobiographic accounts, descriptions and instructions relating to labor and production, and the lyrics of songs. ${ }^{39}$

Une des caractéristiques principales de ce type de textes, en (grande) partie créés par l'ethnographe, c'est le fait qu'ils soient issus de la conversation avec les gens qui l'entourent, des performances ordinaires auxquelles l'ethnographe est appelé à prendre part. Cette nature de textes qu'il appelle "protocolaire", éminemment progressive, est pour le moins éloignée de la notion de textes tels que rassemblés dans un corpus d'étude littéraire. Parlant de la capture vidéo de la pièce de théâtre "Le pouvoir se mange entier » qui constitue l'objet de son essai Power and Performance, il affirme :

It would be arbitrary to isolate one event from the process to which it belongs and to declare the filmed performance the definitive play. For that reason alone a definitive text cannot be exhibited ${ }^{40}$. 
Si ce parti pris fait la part belle aux traces textuelles, fragments de performance (théâtrale ou non) enregistrés, recopiés, consignés par écrit ou collectés, il a deux implications méthodologiques et épistémologiques cruciales. Tout d'abord, il implique de renoncer à l'idée de texte comme entité close et définitive. Par conséquent, il invite à qualifier de textes des supports matérialisés par l'intervention du chercheur, qui ne consistaient auparavant qu'en fragments oraux de discours ou d'interactions, et à en faire des objets d'analyse - au même titre que des textes orthodoxes - à même d'éclairer le contexte général étudié (sociopolitique, sociolinguistique, sociolittéraire) et de mieux percevoir les formes du discours littéraire.

Dans son dernier livre publié en 2008, Ethnography as Commentary. Writing from the Virtual Archive, Johannes Fabian revient sur ce parti pris méthodologique qu'il avait avancé dans les années 1990 et précédentes. Il consacre entièrement cet essai à l'expliquer en justifiant le choix de son objet d'étude : une conversation qu'il eut jadis à Lubumbashi avec Kahenga, un féticheur venu désenvoûter sa maison pour en débarrasser ses occupants de récurrents problèmes d'insécurité. Ce dialogue date de près de quarante ans, à l'époque où l'auteur amorçait son travail anthropologique de longue haleine au Katanga. Ayant enregistré cette conversation, il considère que le commentaire qu'il peut en faire a posteriori constitue une analyse venant non seulement éclairer ladite conversation et enrichir la connaissance sur la médecine traditionnelle en Afrique, mais qui peut plus largement être intégrée à un corpus général de textes ethnographiques patiemment constitué au fil des mois et des années d'enquête; un corpus informant, dans son cas, tant les savoirs populaires que le théâtre populaire et les questions de sociolinguistique en Afrique centrale :

In other words, when ethnography is called text-centered this regards above all its epistemological foundation; it does not mean that collecting texts is the principal purpose of research. A text, to become " collectible ", must be relevant to a project that is always wider than what a specific text may document. This also applies to the document selected for interpretation in this book. [...] Perhaps not when it took place but certainly afterwards, the conversation with Kahenga became part of a corpus of texts, a term that may have a technical meaning in literary theory but never loses completely its metaphorical connotations. Like a body, a corpus has size, volume, weight, articulation of parts and members; as long as it is alive it grows and changes. The latter, growth and change, certainly fit the corpus of documents I produced (more often than found) in the course of my work as an ethnographer ${ }^{41}$.

Non content de défendre une approche herméneutique de l'ethnographie qu'il se refuse à opposer à l'approche dialogique (les deux étant communément présentées comme aux antipodes l'une de l'autre, comme il l'explique p.36-38), Johannes Fabian revendique même la pertinence épistémologique de l'opération consistant à fabriquer son corpus, à faire ses textes :

There is another reason to emphasize "making ». It expresses a position according to which knowledge production involves objectivation. Only subjects can know, yet in order to be presented and shared, subjective experiences and insights must become objectified. They must exist materially, be embodied, in the knowing subject, in the kinds of sensual mediations that enable subjects to communicate through language, and in all the other things and practices we usually call culture. [...] Texts are among the objectivations needed for producing ethnographic knowledge ${ }^{42}$.

Évidemment, l'auteur parle là depuis sa discipline et les méthodes qui lui sont propres, l'anthropologie, et on pourrait alors nous objecter que cela ne saurait concerner les 
études littéraires et théâtrales qui sont censées parler des textes consacrés, d'artefacts clos et signés par un auteur, appartenant à un système littéraire qui en assure la chaîne de diffusion auprès d'un lectorat et d'une masse critique. Mais cela n'est valable que dans des contextes sociolittéraires où la littérature et le théâtre se conçoivent exclusivement comme tels, selon ce modèle hérité de la littérature moderne occidentale, ce régime "d'excellence et de singularité démocratique " dont Nathalie Heinich a établi l'histoire sociale (en analysant le cas, certes particulier, de la France) ${ }^{43}$. Or si la plupart des pays d'Afrique ont hérité de ce puissant modèle (en termes d'influence) inculqué durant la période coloniale, il coexiste avec d'autres façons de faire du texte, de créer de la littérature qui n'impliquent pas nécessairement les mêmes dispositifs de création du texte ni d'accès au public. Même si on s'accorde avec Alain Ricard, qui lisant Soyinka, écrivait qu' " en Afrique aussi, le théâtre est une construction poétique, non un donné anthropologique ${ }^{44}$ ", il reste que la variété des formes de textes rencontrés sur le terrain nous conduit à emprunter d'autres méthodes, à adopter d'autres conceptions du texte, quitte à accepter la possibilité de la co-construction (même partielle) du corpus avec un auteur local et non plus un « informateur " (figure bien connue d'une anthropologie à l'ancienne, source d'inspiration de maints écrivains africains). Ce qui advient alors est un "co-texte», comme l'écrit Fabian, subtil néologisme qui dit tout autant la richesse sémantique contenue dans un texte écrit, souvent transcrit, d'après une performance ou un dialogue et informant le contexte général dans lequel le texte s'inscrit, que la collectivisation de l'opération menée à quatre mains ${ }^{45}$. Dans l'un et l'autre cas, il s'agit d'un texte spécifique à haute valeur ajoutée d'ordre ethnographique, sociolinguistique et littéraire.

31 Lors de ma recherche doctorale au Katanga, la création de "co-textes » fut une étape décisive car constitutive de la composition de mon corpus. Travaillant dans une perspective comparatiste français/swahili, j'avais collecté, par le biais de rencontres avec leurs auteurs ou de visites régulières dans les librairies officielles et officieuses (les « librairies par terre », qui rappellent les bouquinistes ambulants en Europe), des textes théâtraux écrits en français - publiés ou non - ainsi que des textes semi-oraux, c'est-àdire des improvisations en swahili de scènes listées et décrites dans un canevas écrit en français. J'avais alors filmé, transcrit et traduit ces spectacles, avec la précieuse coopération d'un des acteurs d'une des deux troupes théatrales swahiliphones sur lesquelles je travaillais, Sando Marteau de la Troupe Théâtrale Mufwankolo (la même que celle étudiée par Johannes Fabian, dans Power and Performance, voir supra). Les longues discussions qui émergeaient de cette traduction collective participaient à l'écriture de ce co-texte, cet autre texte que devenait la traduction française d'une performance dont les mots n'avaient pas été fixés à l'avance et qui n'était pas destinée à être répétée telle quelle verbalement ; un texte qui en outre était truffé de références culturelles très spécifiques - l'environnement dans lequel le spectacle était joué (du général au particulier: le Congo, le Sud-Katanga minier, la ville de Lubumbashi, le quartier ou la commune dans lequel le spectacle enregistré avait été joué) mais également liées à l'actualité sociopolitique du moment - qu'il nous fallait expliciter ensemble pour prétendre sonder un tant soit peu « l'épaisseur » sémantique du texte. Ce co-texte, étudié en miroir du canevas auquel je pouvais avoir accès, m'a fourni une mine d'informations pour comprendre la configuration et les enjeux sociolinguistiques de la région. Si ces textes devaient un jour être publiés (en l'état, ils ne figurent qu'en annexe de ma thèse), ils nécessiteraient un appareil de notes extrêmement touffu ${ }^{46}$. 


\section{Tisser le texte pour saisir des formes de vie}

constitution, son devenir et ses usages, ainsi que l'intérêt et la richesse d'un corpus hétérodoxe ouvert et vivant, reste que la littéraire que je suis continue de s'interroger, toujours dans une perspective socio-anthropologique, sur la singularité du discours littéraire et de la performance théâtrale par rapport à cette collection de textes et traces collectés et matérialisés ainsi que sur leurs apports spécifiques à l'analyse de ce que Marielle Macé appelle, à la suite de Wittgenstein et Stanley Cavell, des «formes de vies » ${ }^{47}$. Cherchant à percevoir «l'effet-littérature » dans une économie générale de discours (ce que Marc Angenot qualifiait de « système socio-discursif global » ${ }^{48}$ ), je m'en remets donc à l'auteure de Styles. Critique de nos formes de vies, qui cherche d'une certaine manière à répondre à cette question :

[...] la littérature (en particulier la moderne) m'apparaît comme le lieu principal où s'est affûtée cette attention au caractère stylistique de la vie elle-même, au pluriel des styles, des façons d'habiter les formes et de leur donner sens. La littérature assume cette tâche d'explicitation de valeurs jusque dans ses contradictions et ses incertitudes, les textes littéraires, dans leur variété et leur singularité même, constituant le lieu par excellence où se médite sans se fixer le sens du « comment ». [...] Il y a en fait dans l'écriture et la façon dont on la reçoit une morale en acte du formel de la vie ${ }^{49}$.

Considérer les formes de vies comme des styles implique de faire une anthropologie des formes ou des manières de faire et de vivre, conçues par l'anthropologue François Laplantine comme autant d'actes de créations :

Les formes ne sont pas, comme on le dit souvent, des "expressions" mais des créations. [...] La notion d'expression (sociale, psychique, linguistique) suppose l'existence déjà là de signifiés en attente de signifiants. Formes, modes, manières, kata sont des créations, ce sont des actes ${ }^{50}$.

Et l'auteur d'ajouter qu'il s'intéressera par conséquent dans cet essai aux créations artistiques et à leurs auteurs. Il s'agit donc, dans son acception comme dans celle de Marielle Macé, de prêter attention à l'ensemble des manières d'être, de faire, de parler, de poser une parole publique, une approche qui devrait permettre de discerner l'endroit (les endroits) de la forge de la parole poétique et fictionnelle, de percevoir les enjeux de son surgissement et les caractéristiques de sa mise en forme dans telle situation donnée.

\section{«Penser le sensible » : percevoir le grain de son terrain et l'ombre de sa présence}

Reprenons la lecture de Faire Anthropologie, Art, Archéologie et Architecture de Tim Ingold. L'ensemble de son essai s'articule autour du cours d'anthropologie qu'il donna à l'Université d'Aberdeen, intitulé «les $4 \mathrm{~A}$ », dont le premier objectif était de percevoir les «matériaux de la vie $»^{51}$ non seulement par l'intellect mais aussi et d'abord par les sens. Il s'agissait dans un premier temps de "toucher les objets", ramassés par les étudiants autour de "la chose» située en un lieu de leur choix, choisie par chacun comme cas d'étude personnel. Ces objets du quotidien (trombones, canettes vides, pièces de monnaie, plumes, balles en caoutchouc...) devaient être conservés comme tels et manipulés avec précaution, comme des objets «qui portaient témoignage d'autres 
vies » et qui pourtant "avaient rompu avec ces vies » ${ }^{52}$, lesquels objets, pourrait-on poursuivre, étaient désormais rendus disponibles pour de nouvelles vies inaugurées par la rencontre avec les étudiants qui les avaient ramassés. Dans un second temps, les étudiants étaient invités à "sentir les matériaux » :

Avec les matériaux, au contraire, l'expérience tactile portait exclusivement sur le grain et la texture, sur la sensation de toucher une substance malléable avec une peau sensible, que ce soit du sable versé sur la paume et glissant entre les doigts, ou de la terre mouillée, collante, se transformant en croûtes lorsqu'elle sèche, ou encore les éraflures provoquées par le gravier, et ainsi de suite ${ }^{53}$.

Cette méthode d'appréhension sensible des lieux et des choses est, pour Tim Ingold, à la base du travail de terrain qu'est l'observation participante en anthropologie. Je crois en effet qu'une approche sensible des lieux que nous traversons et habitons pour nos recherches et des choses (en l'occurrence des textes) que nous rencontrons sur notre chemin peut orienter singulièrement notre réflexion vers des facettes de formes de vie qui, sans elle, seraient demeurées cachées. "Penser le sensible » comme nous y invite François Laplantine quand on fait (au sens plein du mot) un terrain littéraire et théâtral, c'est être attentive à toutes les dimensions du vécu, rester éveillée au grain de la vie quotidienne dans ses aspects les plus ordinaires et, concomitamment, dans une dialectique d'équilibriste, de maintenir le regard vers soi, se percevoir soi dans cet environnement où l'on a choisi de venir s'installer temporairement pour tâcher d'en comprendre, via les créations et leurs auteurs rencontrés, les formes de vie, les manières de traiter du texte, de la performance et de l'art. Se percevoir soi non par narcissisme déplacé mais dans l'optique d'identifier les effets provoqués - sur ses interlocuteurs, voire sur le champ étudié - par sa propre présence dans l'environnement choisi qu'est le terrain d'étude.

\section{«Penser le sensible », le sensoriel, les ambiances, les affects}

Penser le sensible consiste donc dans un premier temps à porter une attention particulière à l'ambiance d'un lieu, prendre le temps de la sentir et de se laisser envahir par elle. Si, comme le précise l'historien Didier Nativel, dans le chapitre 8 de sa thèse d'HDR consacré à «la ville comme déploiement du sensible», l'ambiance n'est pas encore un objet académique complètement institué, certains auteurs (dont lui) le travaillent pourtant, y voyant un puissant réservoir de sens (tout à la fois sensoriels et sémantiques) ${ }^{54}$. François Laplantine dit d'elle qu'elle est « une notion transversale ou plutôt traversière, qui traverse les disciplines » et qu'« en elle, s'effectue une rencontre entre un milieu et un moment, c'est-à-dire un espace et un temps $\aleph^{55}$. C'est également en ces termes que Didier Nativel conçoit son approche de l'ambiance d'après sa lecture de Trois Orients de Claudio Magris :

[...] j'avais été touché, et le terme n'est pas anodin, par des remarques de Claudio Magris sur la relation voyageur/paysage. Il explique qu'un paysage est "stratification de terre et d'histoire», constitué de "sentiers, de maisons, de roches" mais avant tout de «sociétés, de personnes, de gestes, d'habitudes", de passions... c'est donc particulièrement le lieu du mouvant. D'autant, ajoute Magris, que la relation au paysage est souvent dynamique et singulière : on le parcourt en suivant son rythme, sa personnalité, son histoire en effectuant sa propre lecture. Ce qui est dit des étrangers, des observateurs, peut l'être aussi des usagers ordinaires de la ville qui oscillent en permanence entre décryptage et coproduction des paysages et ambiances qui se dégagent d'une ville ${ }^{56}$. 
Cette "coproduction des paysages et ambiances » correspond à l'expérience que Laplantine appelle, après Rancière, "le partage du sensible ", c'est-à-dire le type d'interaction que nous pouvons avoir avec une ambiance :

Une ambiance ne peut être individuelle. Elle est toujours ambiance commune d'une scène dans laquelle nous nous trouvons, ce qui suppose un partage du sensible. [...] De cette première approche, trois notions me semblent pouvoir être retenues : un lieu commun, un temps commun, un lieu qui crée du lien ${ }^{57}$.

Et c'est aussi sur la nature précise de ce lien entre soi et l'ambiance de l'environnement choisi, que va se porter la réflexion de celles et ceux qui cherchent à capter une ambiance et à en faire ressortir un savoir sur les formes de vies auxquelles elle renvoie. S'atteler à une telle radiographie des ambiances urbaines africaines nécessite pour Didier Nativel une rigueur d'autant plus difficile à tenir qu'elle exige du chercheur qu'il trouve le juste positionnement dans ce subtil balancement "entre distanciation et empathie avec les témoins que l'on interroge et les lieux ", ce qui nécessite " une prise de conscience socio-historique (Noiriel, 2006) pour éviter une dérive "présentiste" de projection incontrôlée des affects et des thématiques contemporaines sur des réalités sociales passées ${ }^{58}$. Certain.e.s anthropologues, qui, de par la nature même de leur discipline, sont déjà acquis.es à la cause d'une approche sensible des faits sociaux, assument largement ce risque, en n'hésitant pas à user d'une écriture sensorielle de leurs expériences personnelles informant leurs affects dans la rencontre avec le milieu étudié. Ainsi en est-il par exemple du texte "Granite» de l'anthropologue américaine Kathleen Stewart dans lequel elle raconte comment la sensation du granit sur sa peau nue, une nuit, au retour d'une soirée bien arrosée, produisit immédiatement une impression de « end-of-life attunement " (l'impression de voir sa vie défiler et tout son savoir se dissoudre, à l'exception du paysage granitique dans lequel elle évoluait) qui la conduisit à une réflexion, empreinte de son expérience personnelle, sur l'histoire sociale du granit au Nord-Est des États-Unis où elle grandit ${ }^{59}$. Ce que Kathleen Stewart relate dans ce petit texte, c'est une expérience de «densification du présent » établie par Clifford Geerz et rappelée par Laplantine ${ }^{60}$ ou encore par une autre anthropologue américaine, Anna Tsing dans son dernier essai, Le Champignon de la fin du monde ${ }^{61}$.

Ainsi, dans la perspective de Kathleen Stewart, on est autant fait par son terrain, façonné par lui, qu'on le fait, ainsi que le veut l'expression consacrée "faire un terrain ». C'est également ce que défend Aurore Vermylen qui revendique les « apports de l'auto-analyse " comme une méthode de compréhension du terrain d'étude. Elle choisit d'être à l'écoute des émotions qui jaillissent sur son terrain dans les Grands Lacs et de les analyser en contexte pour tenter de comprendre différemment - via les sens et l'émotionnel donc - les émotions qui façonnent le vécu des habitants de la région ${ }^{62}$.

Finalement, ce contre quoi Didier Nativel mettait en garde et que ces anthropologues n'hésitent pas à pratiquer, cette «densification du présent " qu'il qualifie d'après Hartog, de "dérive présentiste », n'est-ce pas ni plus ni moins la matière-même de la littérature? N'est-ce pas là l'expression de sa capacité à s'étendre sur le vécu, l'émotion, la sensation, la part sensible de la vie pour en qualifier au mieux ses formes et tâcher d'en proposer une interprétation? L'analogie entre une certaine ethnographie et la littérature est un débat ancien auquel Clifford Geertz a déjà proposé une synthèse (L'anthropologue comme auteur) mais la question qui se pose à nous qui étudions la littérature et la performance, en partie à la manière des anthropologues, est la suivante: en quoi cette façon littéraire d'aborder et d'écrire son terrain d'étude, 
offre-t-elle des clés pour «trouver » les textes sur nos terrains littéraires et théâtraux, pour les manier et les « faire parler»?

41 Il me semble que ce partage du sensible opéré par le chercheur sur son terrain lui permet non seulement de repenser ses présupposés de recherche, de ré-orienter ses intuitions premières et de dessiner, avec ses interlocuteurs une cartographie de sa recherche qui en dira davantage sur son objet que n'importe quel corpus prédéfini à l'avance dans un texte à la forme déjà fixée par la publication et canonisée par l'histoire littéraire. Ainsi, après ses propres déambulations dans la ville informées par sa propre cartographie mentale de l'organisation littéraire nairobienne, Olivier Marcel a fait dessiner à deux écrivain.e.s et performeur.e.s contemporain.e.s nairobien.ne.s (Tony Mochama et Ngwatilo Mawiyoo), les cartes mentales des pratiques littéraires à Nairobi. Adjointes à un long travail d'observation participante à Nairobi, elles lui ont permis de dessiner une cartographie actualisée de la vie littéraire kenyane révélant que le centre littéraire historique où s'est fondée l'histoire de la littérature moderne kenyane dans les années 1950-1960, n'était plus si central dans le Nairobi du début des années $2000^{63}$.

Une approche sensible des lieux parcourus et habités par le/la chercheur.e mobilise donc chez lui/elle des capacités d'observation accrues et surtout une certaine souplesse dans l'appréhension de son objet d'étude susceptible de le/la conduire à reconfigurer le paysage littéraire et, partant, à envisager sous un autre œil les dynamiques sociales, sociolinguistiques et politiques qui l'innervent. Il s'agit donc d'une part de prêter une attention particulière à la fois aux lieux réels, concrets, tangibles où s'organise l'activité littéraire et théâtrale (à leurs usages, aux façons qu'ont les auteurs de les habiter, de les investir, voire de les travestir), mais aussi, d'autre part, à la façon dont les espaces partagés qui fondent le socle de l'identité d'une ville et d'une région sont pensés par leurs auteurs.

À titre d'exemple, l'orientation régionale "Grands Lacs » ${ }^{64}$ que j'ai donnée à ma recherche actuelle est au moins autant un parti pris de départ (d'après le constat que l'histoire et la vie sociopolitique de ces régions/pays sont complètement intriquées) qu'une prise en compte du fait que cette région constitue un topos littéraire, un paysage imaginaire tissé de toutes parts par les auteurs de la région. Cette observation est venue corroborer une intuition de recherche avancée dans un article sur la "Géocritique de la RDC » dans lequel j'identifiais deux topoï littéraires majeurs du Congo, le fleuve Congo et l'Est du pays présenté comme une interface avec l'Est du continent, un espace éminemment transfrontalier connecté aux pays voisins ${ }^{65}$. Cette intuition se fondait sur des textes écrits par des auteurs congolais, pour la plupart kivutiens (originaires et/ou vivant encore au Nord-Kivu ou au Sud-Kivu), mais qui étaient majoritairement publiés et diffusés par le principal organe du livre au Congo, les maisons d'édition et librairies Médiaspaul et les Paulines (anciennement Saint-PaulAfrique, de la congrégation des Paulinien.ne.s), fort bien implantées dans le pays. Aussi avais-je trouvé ces petits romans se déroulant au Kivu à la librairie Médiaspaul de Lubumbashi au Katanga et c'est lors de mes terrains ultérieurs au Kivu, au gré de ma lecture des textes "trouvés » sur place à Goma et Bukavu, que j'ai pu valider cette hypothèse de l'Est et plus largement des Grands Lacs un topos littéraire. Qu'ils écrivent depuis le Rwanda, le Burundi ou l'Est de la RDC, les auteurs ont une conscience aiguë de la puissance imaginaire et de la richesse poétique de cet espace qu'ils évoquent, décrivent ou chantent dans leurs textes. Les paysages extraordinaires de la région sont fréquemment convoqués comme métaphores, la puissance des éléments naturels la 
façonnant (tels que les lacs, les collines et les volcans) servant à exprimer l'intensité des émotions qui y règnent et la déstabilisent ainsi que le saisissant paradoxe d'une terrible beauté. La forêt, communément appelée " pori » (littéralement la brousse en swahili), qui recouvre une part importante de la région, est présentée comme le lieu de tous les dangers, son caractère sauvage de nature non domestiquée étant synonyme de chaos, de violence et de guerre. En effet, c'est dans la forêt que se cachent les milices et groupes armés qui terrorisent la région, c'est aussi là que se réfugient régulièrement les habitants persécutés, chassés de chez eux par les groupes armés.

L'attention au paysage comme trait d'union de cet espace régional transfrontalier non seulement m'invite à l'envisager à cette échelle et dans une perspective circulatoire et réticulaire (en m'intéressant à l'organisation et à la circulation spatiale des textes de théâtre comme des motifs qui y sont déroulés ainsi qu'à leurs usages) mais permet aussi de laisser jaillir de soi des textes "trouvés » et parfois sollicités par le/la chercheur.e dans le cadre de conversations avec ses interlocuteurs. Ainsi Johannes Fabian racontet-il comment la Troupe Théâtrale Mufwankolo (de Lubumbashi) imagina, de son plein gré mais après une conversation qu'il eut avec eux, un spectacle à partir du proverbe à propos duquel il avait partagé avec elle ses questionnements (« Le pouvoir se mange entier $)^{66}$. Plus récemment, Jean-Baptiste Lanne, dans sa thèse de doctorat en géographie, assume également de baser partiellement son analyse du vécu des acteurs quotidiens de la sécurité à Nairobi (la perception de soi, leur mode d'habiter la ville, leur mode d'appréhension de la temporalité singulière de la "veille » et d'une vie dans l'attente) sur des poèmes qu'il leur a demandé d'écrire dans l'idée de contrer «l'atonie narrative ${ }^{{ }^{67}}$ ressortant des récits de vie classiques qu'il avait commencé par solliciter. Même si son objectif n'était initialement pas de faire une analyse littéraire, c'est l'exploitation du poétique des propos de ses interlocuteurs qui lui permit d'accéder aux perceptions personnelles qu'il cherchait à comprendre chez ces acteurs de la sécurité. Il s'attachait dans la conversation qu'il avait avec eux à relever une "petite bizarrerie $\aleph^{68}$ de langage (parfois et souvent due au fait que l'anglais n'était pas la langue première de ses interlocuteurs) et les invitait à développer cette parole à potentiel poétique, à « déplier leurs formules " $^{69}$ dans un poème qu'ils écrivaient alors à ses côtés et avec son concours, dans un échange conversationnel.

\section{Percevoir l'ombre de sa propre présence}

Lorsqu'il prit conscience de la richesse qu'offrait la pratique poétique pour éclairer ses questionnements, Jean-Baptiste Lanne décida de l'instituer comme méthode de travail, ce qui impliqua d'instaurer un véritable dispositif d'écriture. Il raconte alors la façon dont il ritualisa cette activité. La dimension répétitive qu'il institua ainsi avait deux visées principales : tout d'abord, en faisant de l'écriture du poème un travail fait de corrections et retouches, elle permit aux écrivants de se sentir de plus en plus à l'aise avec cette activité d'une séance à l'autre et donc de libérer progressivement leur parole personnelle, voire de déployer leur style propre ; mais elle a aussi été pensée comme un moyen de rétablir un léger équilibre dans une relation foncièrement dissymétrique puisque c'est lui qui avait la liberté de venir à eux et de les solliciter, eux étant cantonnés à leur poste de travail. ${ }^{70}$ sensible de la ville, Jean-Baptiste Lanne ne se contente pas d'assumer les effets de sa 
présence dans l'environnement parmi ceux qu'il est venu étudier, il s'en sert pour sa recherche. Ce sont le mode et le fruit de sa rencontre avec les askaris qui constituent en soi son analyse du milieu.

Sans surprise, ce questionnement réflexif, qui est le propre de l'anthropologie, se retrouve aussi dans les études théâtrales qui mettent en présence les artistes étudiés et le/la chercheur.e étudiant un processus de création. La part du spectateur dans la création est une question centrale dans l'article de la généticienne Almuth Grésillon qui se demande si, puisque « dans le cas du théâtre, il y a imbrication étroite entre texte et mise en scène, n'y a-t-il pas aussi et nécessairement une partie de la genèse qui englobe déjà le spectateur $?^{71} »$. À cette question rhétorique, on ne peut qu'approuver et reconnaître avec elle qu'il existe bien "des points où production et réception se touchent ${ }^{72}$ », a fortiori dans les spectacles mouvants évoqués précédemment, ces spectacles en perpétuelle recomposition caractérisés par la part importante accordée à l'improvisation.

Ce constat la conduit à pointer l'incomplétude fondamentale du texte dramatique dans l'économie générale du théâtre (eu égard aux apports du travail de plateau) et à considérer qu'il s'agit d'une donnée majeure à prendre en compte dans les études théâtrales. Elle y voit une difficulté inhérente à l'étude du théâtre qui ne se baserait que sur les textes, parfois sur les représentations associées aux textes. Le théâtre étant, plus que les autres créations littéraires, un processus vivant et évolutif, il est d'après elle capital d'assister aux répétitions pour être témoin des différentes étapes de la création et avoir accès aux traces qui les inscrivent matériellement dans le spectacle (notes et carnets de mise en scène). Sont donc ici soulignés non seulement l'apport de la génétique dans les études théâtrales mais surtout la nécessité de la participation du/de la chercheur.e qui doit se faire spectateur/trice de la création en cours dès le début du processus et qui dès lors partage avec l'équipe une expérience commune.

Or le/la chercheur.e n'est pas un.e spectateur/trice ordinaire, il/elle peut être appelé.e à intervenir dans le processus de création. J'en ai fait l'expérience dans le cadre d'une observation de préparation et de représentation d'un spectacle, Vita siyo muchezo ya watoto en swahili (La guerre n'est pas un jeu d'enfants), de Théâtre \& Réconciliation (voir supra) à Goma en juin 2019. Dès mon arrivée, j'ai été invitée, par la metteuse en scène Frédérique Lecomte, par son assistante à la mise en scène comme par plusieurs comédien.ne.s, à donner mon avis sur le travail en cours. En somme, on sollicitait mon « expertise » en tant que connaisseuse du travail et de la méthode de T\&R mais surtout en tant que connaisseuse - swahiliphone de surcroît - du contexte traité dans la pièce. Je me suis prêtée au jeu en acceptant d'endosser ce "second rôle" d'« experte culturelle et dramaturge » que l'on m'assignait ${ }^{73}$ et ai ainsi formulé, d'une répétition et d'une représentation à l'autre, un certain nombre de remarques, critiques, observations et suggestions dont certaines ont été entendues et ont participé à (ré)orienter le spectacle en fonction des objectifs poursuivis. Ainsi, par exemple, ce sont en grande partie mes remarques qui ont conduit Frédérique Lecomte, en accord avec son assistante et l'ensemble de la troupe, à clarifier la distribution, entre Noirs et Blancs, des responsabilités dans l'exploitation du Congo afin d'éviter les malentendus, voire les quiproquos qui dominaient les réactions du public lors des premières représentations. Le spectacle ainsi actualisé et ré-orienté, les représentations qui s'en suivirent se déroulèrent plus calmement et furent reçues avec plus de bienveillance par le public. 

n'en reste pas moins que le fait de constater les effets sur la création étudiée de sa propre intervention est particulièrement troublant pour la chercheuse qui a eu pour habitude de ne considérer comme matière à étudier que ce qu'elle trouve sur son terrain, c'est-à-dire ce qui s'y trouvait déjà avant son arrivée, ce qui s'offre à ses yeux sans qu'elle en soit responsable en aucune manière. Un/e chercheur.e n'est pas censé.e étudier ce qu'il/elle a participé à forger, fût-ce pour le bien du spectacle, le bien-être de la troupe ou du public ; cela n'est pas son affaire. C'est du moins ce que nous a enseigné une certaine épistémologie positiviste des sciences humaines en accord avec le respect de l'objectivité comme valeur cardinale et absolue, censé guider tout travail scientifique.

Cependant, est-il vraiment réaliste (et honnête) d'envisager aborder une situation de manière neutre, c'est-à-dire la considérer comme intacte par essence et devant nécessairement rester hors d'atteinte de nos interprétations faites d'après notre éducation, nos projections et affects? Qu'il soit absolument nécessaire d'avoir conscience de la situation d'où l'on parle en tant que chercheur.e analysant un contexte aux codes étrangers aux siens et de tâcher de limiter la part de ces affects et projections dans le résultat de l'analyse, est incontestable. Mais on peut aussi considérer l'observation participante avec plus de souplesse et de réflexivité, à l'image de nombreux anthropologues qui se sont déjà débarrassés de cet encombrant idéal d'une objectivité flirtant avec un fantasme - tout aussi dangereux dans les dérives auxquelles il peut donner cours - celui de l'authenticité d'un terrain qui serait vierge par essence. Johannes Fabian épingle ce travers sans complaisance dans Power and Performance dans lequel il relate sa propre expérience - éminemment performative - avec les comédiens de la Troupe Théâtrale Mufwankolo:

In ethnographies that pretend to give naked texts or data, nakedness is but another costume. As a writer, the anthropologist fashions and forms his material. He differs from the writer of fiction, not in what he presents, but in what he needs to justify his presentations as contributions to a body of language ${ }^{74}$.

Et de nous rappeler que le rôle de l'ethnographe est un "catalyseur", un "producteur " au sens théâtral du terme, dans le sillage de "l'ethnodramaturge" théorisé par Victor Turner pour qui la performance par le chercheur d'un aspect d'un fait social lui permet d'accéder à une compréhension de ce fait social différente de celle mobilisée par la méthode logique de déduction d'observations et d'analyse de résultats ${ }^{75}$.

53 S'agissant de ma propre implication dans ce spectacle de Théâtre \& Réconciliation, je songe pour le moment à m'inspirer pour mon analyse de terrain de ce second rôle qu'on m'a fait jouer quand je pensais n'en jouer aucun, m'attendant à ce qu'on me laisse dans celui, plus confortable mais illusoire, de l'observatrice silencieuse du hors-jeu. Je m'en inspirerai à différents niveaux. Au niveau de l'analyse du spectacle en lui-même, ma compréhension des implications du choix de tel ou tel propos/expression/ représentation scénique, ma perception fine de la réception du public suivie de mes hypothèses explicatives partagées avec la troupe, sont des apports ayant contribué à ré-orienter le spectacle ; par conséquent, ils constituent autant de « couches » de sens à confronter avec les choix et actes posés successivement durant tout le processus, pour rendre toute la complexité de cette tournée théâtrale mobilisant des acteurs sociaux et de théâtre d'horizons aussi divers, autour d'objectifs et d'ambitions qui peinèrent à se rencontrer réellement. D'un point de vue plus général, je pense que cette prise de 
conscience, qui m'est apparue de manière plus évidente à cette occasion, devra m'inviter à repenser mes interactions avec tous mes interlocuteurs sur le terrain au prisme de ce second rôle qui n'est jamais très loin. Il faut donc apprendre à les deviner en étant très attentive aux interactions avec les interlocuteurs (savoir entendre les non-dits et allusions) et aussi tâcher de percevoir la manière dont ils (se) représentent l'effet de notre présence dans leur environnement, dont ils rendent visibles la façon dont ils vivent (ou veulent vivre) leur relation à nous, mais aussi la façon dont, sans toujours nous solliciter, ils intègrent "quelque chose de nous » dans leurs textes : une idée, un propos, une forme, des expressions qui sont venues des discussions que nous avons eues avec eux et elles.

\section{Conclusion}

Du chiffonnier à l'anthropologue, voire à l'ethnodramaturge ? C'est en tout cas le mode d'action que prône Bernard Müller qui revendique ce terme et la méthode afférente consistant à " performer l'ethnographie ", forgée par Victor Turner ${ }^{76}$ et reprise à son compte par Johannes Fabian. S'appuyant sur son expérience avec une troupe de théâtre yoruba au Nigeria au cours de laquelle il se retrouva à occuper le rôle de dramaturge qu'on lui assignait (expérience analogue à la mienne, avec Théâtre \& Réconciliation à Goma, voir supra), il en vient à assumer cette " casquette ", à se l'approprier en étayant l'argument de la collusion entre l'ethnographe et le dramaturge, jusqu'au point de produire un spectacle (et d'en être le dramaturge) portant sur le phénomène qu'il étudie, la concomitance de l'émergence de la conscience d'une africanité brésilienne et d'une brésilianité africaine ${ }^{77}$. Si tel n'est pas tout à fait mon credo, il reste que l'analogie entre recherche théâtrale et anthropologie, entre ethnographe et dramaturge, est inspirante, dans la mesure où elle nous invite à revoir notre acception de ce qu'est ou ce qui fait texte de théâtre. Ce faisant, elle nous conduit à considérer les traces matérielles - pièces de théâtre, fragments scripturaires, notes et carnets de création comme des éléments parmi d'autres du corpus d'analyse que nous participons activement à créer, bien au-delà des opérations ordinaires/orthodoxes de collecte et d'assemblage.

Si l'objectif de cet article était d'interroger le nœud conceptuel et méthodologique entre théâtre, archives et génétique textuelle, c'est depuis mon terrain, où je me livrai déjà à des réflexions d'ordre méthodologique et réflexif, que j'ai pensé cet article. Même pour une recherche historienne, c'est aussi le terrain qui permet d'orienter une recherche dans les archives, fussent-elles conservées en Europe. Lire l'histoire coloniale du Congo ne m'informera que très partiellement des dynamiques théâtrales à l'œuvre à l'époque, des lieux et cercles de sociabilité artistique. En me rendant sur place, je peux échanger avec des interlocuteurs qui me donneront des informations susceptibles de m'aider à m'orienter plus efficacement dans les archives, je peux aussi apprendre, via ma propre expérience ou ma pratique du lieu, à percevoir son organisation tant spatiale que sociale, ce qui me permettra de restituer plus finement les dynamiques sociales et politiques du théâtre qui l'ont innervé jusqu'à aujourd'hui.

Ce fut donc mon expérience - alors particulièrement dense - du terrain qui m'amena à ces questionnements. En effet, la recherche que je mène actuellement me confirme le rôle absolument central et déterminant du terrain qui était déjà le point de départ de ma recherche doctorale mais dont je n'avais, à l'époque, pas autant saisi la richesse, le 
fort potentiel d'analyse et de compréhension du sujet traité et du terrain choisi en général. Bien au-delà de la collecte d'informations et de textes ou d'une meilleure compréhension du contexte sociopolitique d'où émergent les textes, le terrain est une véritable rencontre dont il est important de bien connaître les acteurs pour en saisir toute la portée : il s'agit d'une rencontre entre soi et un (mi)lieu. S'il est clair que l'objet de notre venue sur ce terrain est de contribuer à la connaissance et à la compréhension de ce qu'il s'y passe - en l'occurrence dans mon cas, ce qu'il s'y joue, au double sens du terme, dans les écrits et sur la scène - il m'apparaît plus que jamais que la connaissance de soi et la perception aiguë des effets de sa propre présence sur ce terrain, devraient informer l'analyse. Dès lors que les textes collectés sont essentiellement le fruit de rencontres avec leurs auteurs, le second rôle assigné au chercheur (parfois à son insu) doit être non seulement accepté mais pris en compte dans l'étude des matériaux textuels.

\section{BIBLIOGRAPHIE}

AGIER, Michel, « Le tournant contemporain de l'anthropologie », Socio, 2013, n 1, « Penser global », p. 77-93.

ANGENOT, Marc, « Que peut la littérature ? Sociocritique littéraire et critique du discours social » dans NEEFS, Jacques \& ROPARS, Marie-Claire (éd.), La Politique du texte. Enjeux sociocritiques. Pour Claude Duchet, Lille, Presses universitaires de Lille, 1992.

BARBER, Karin, Africa's Hidden Histories. Everyday Literacy and Making the Mind, Indianapolis, Indiana University Press, 2006.

BARBER, Karin, The Anthropology of Texts, Persons and Publics. The Oral and the Written in Africa, Cambridge, Cambridge University Press, 2007.

CLIFFORD, James et MARCUS, George (éd.), Writing Culture: the Poetics and Politics of Ethnography, University of California Press, 1986.

CLIFFORD, James, The Predicament of Culture: Twentieth-Century Ethnography, Literature, and Art, Harvard University Press, 1988.

DUCOURNAU, Claire, La Fabrique des classiques africains. Écrivains d'Afrique subsaharienne francophone (1960-2012), Paris, CNRS éditions, 2017.

FABIAN, Johannes, Ethnography as Commentary. Writing from the Virtual Archive, Durham \& London, Duke University Press, 2008.

FABIAN, Johannes, Power and Performance. Ethnographic Explorations through Proverbial Wisdom and Theater in Shaba, Zaïre, Madison, University of Wisconsin Press, 1990.

FASSIN, Didier, « The ethical turn in Anthropology. Promises and uncertainties », HAU. Journal of Ethnographic Theory, vol. 4, n 1, 2014, p. 429-435.

FÉRAL, Josette, «Introduction: Toward a genetic study of performance - Take 2 », Theatre

Research International, vol. 33, n³ 3, 2008, p. 223-233, p. 225.

FOUÉRÉ, Marie-Aude, «Engagez-vous, rengagez-vous dans les études sur le swahili

aujourd'hui ! », Cahiers d'Études africaines, LV (3), 219, 2015, p. 587-601.

GAHUNGU, Céline, « Le Concours théâtral interafricain : quelles archives ? Quels usages ? ", Continents Manuscrits, $\mathrm{n}^{\circ} 13$ "Construire les mémoires des théâtres d'Afrique : de la trace à

l'archive », URL : https://journals.openedition.org/coma/4210. 
GEERTZ, Clifford, Ici et là-bas. L'anthropologue comme auteur, [1988], Paris, Métailié, 1992. GRÉSILLON, Almuth, « De l'écriture du texte de théâtre à la mise en scène », Cahiers de praxématique, $\mathrm{n}^{\circ} 26$, «Les mots et la scène », 1996, document 4, mis en ligne le $1^{\mathrm{er}}$ janvier 2015 , consulté le 4 décembre 2018. URL : http://journals.openedition.org/praxematique/2979 INGOLD, Tim, Faire anthropologie, archéologie, art et architecture [2013]. Traduit de l'anglais par Hervé Gosselin et Hicham-Stéphane Afeissa, éditions Dehors, 2018.

INGOLD, Tim, Marcher avec les dragons. Paris, Zones sensibles, 2013 et Paris, Le Seuil, coll. « Points », 2018.

LANNE, Jean-Baptiste, « Des vies en veille. Géographies abandonnées des acteurs quotidiens de la sécurité à Nairobi ». Thèse de doctorat en géographie soutenue à l'Université de BordeauxMontaigne, sous la co-direction de Bernard Calas et Myriam Houssay-Holzschuch, 28 novembre 2018.

LAPLANTINE, François, Penser le sensible, Paris, Pocket, coll. « Agora », 2018

LAURENT, Pierre-Joseph, Devenir anthropologue dans le monde d'aujourd'hui, Paris, Karthala, coll. « 4 vents », 2019.

LE LAY, Maëline, « Géocritique de la RDC », dans DIANDUE Bi Kacou Parfait (éd.), « Une géocritique de l'Afrique. Mutations et stabilité de la spatialité et de la temporalité dans le locus africain », Revue Baobab (Université Cocody d'Abidjan), 2009.

LE LAY, Maëline, « Humanitarian Theatre in the Great Lakes Region : In Pursuit of Performativity ", dans NDALIKO, Chérie Rivers \& ANDERSON, Samuel Mark, eds, The Art of Emergency. Aesthetics and Aid in African Crises, Oxford \& New York, Oxford University Press, à paraître en décembre 2019.

LE LAY, Maëline \& ORBAN, Jean-Pierre (dir.), « Écrire le Fleuve Congo après Conrad », Continents manuscrits [En ligne], $\mathrm{n}^{\circ}$ 11, 2018.

LECOMTE, Frédérique, dir., Théâtre \& Réconciliation. Méthode pour une pratique théâtrale dans une zone de conflit, Bruxelles, La Lettre volée, coll. « Essais », 2016.

MACÉ, Marielle, Styles. Critique de nos formes de vies, Paris, Gallimard, coll. « nrf essais », 2016. MARCEL, Olivier, « Le Nairobi des poètes : une cartographie des pratiques littéraires de la génération Kwani? ", Études Littéraires Africaines, « Nairobi », n 31, 2011.

MCAULEY, Gay, « Not Magic but Work. Rehearsal and the Production of Meaning », Theatre Research International, vol. 33, $\mathrm{n}^{\circ}$ 3, 2008, p. 276-288.

MÜLLER, Bernard, « Le terrain : un théâtre anthropologique », dans BIET Christian et ROQUES Sylvie (dir.), Communications, « Performance. Le corps exposé », n 92, 2013, p. 75-83.

MÜLLER, Bernard, La tradition mise en jeu. Une anthropologie du théâtre yoruba, La Courneuve, éditions Aux lieux d'être, coll. « Mondes contemporains », 2006.

NATIVEL, Didier, «Les sens de la nuit. Enquête sur les sensorialités urbaines coloniales à Madagascar et au Mozambique ", Sociétés politiques comparées. Revue européenne d'analyse des sociétés politiques [En ligne], dossier « Nuits urbaines », n³8, janvier-avril 2016.

NATIVEL, Didier, chap. 8 , "La ville comme déploiement du sensible ", Contribution à une histoire des sociétés et des espaces urbains de l'océan Indienoccidental (xixe-xxe), HDR d'histoire, Université Paris-Diderot Paris 7, 2013.

RICARD, Alain, « Vertus de l'indiscipline : langues, textes et traductions », discours d'ouverture des $4^{\text {es }}$ Rencontres des Études Africaines en France (REAF), 5 juillet 2016, Études Littéraires Africaines, $n^{\circ}$ 42, 2016, p. 107-124.

RICARD, Alain, BARBER, Karin et COLLINS, John, West African Popular Theatre, Bloomington/ Indianapolis, James Currey/Indiana University Press, 1997.

RICARD, Alain, Le Principe d'Asihu, 51', CNRS images [1982], 2011.

RICARD, Alain, Littératures d'Afrique noire. Des langues aux livres, Paris, CNRS éditions/Karthala, 1995.

RICARD, Alain, Texte moyen, texte vulgaire. Essai sur l'écriture en situation de diglossie. L'écrivain public 
Félix Couchoro et les comédiens ambulants du Happy Star Concert. Lomé, Togo. 1962-1977, Bordeaux, thèse de doctorat, Bordeaux III, sous la direction de Robert Escarpit, 1981.

RICARD, Alain, Wole Soyinka et Nestor Zinsou. De la scène à l'espace public. Religion et politique, Paris, Karthala, coll. « Lettres du sud », 2015.

STEWART, Kathleen, « Granite », Society for Cultural Anthropology, for the series "Correspondance", février 2018, 2019, URL : https://culanth.org/fieldsights/granite STEWART, Kathleen, Ordinary Affects, Durham \& London, Duke University Press, 2007.

TSING, Anna, Le Champignon de la fin du monde. Sur la possibilité de vivre dans les ruines du capitalisme, préface d'Isabelle Stengers, traduit par Philippe Pignarre, Paris, La Découverte, 2017.

VERMYLEN, Aurore, « Par-delà les émotions et la raison. Les apports de l'auto-analyse comme méthode de compréhension de nos terrains ", revue e-Migrinter, [En ligne], n 18, 2019, mis en ligne le 11 septembre 2019, consulté le 30 septembre 2019. URL : http://journals.openedition.org/ e-migrinter/1781

\section{ANNEXES}

\section{Notes prises durant un des spectacles de Théâtre \& Réconciliation à Goma}

Entre crochets figurent les commentaires hors-jeu : ceux du Joker du spectacle, de la metteuse en scène et du public. Surlignés en jaune sont les passages indiquant un changement notable de mise en scène à l'intérieur des scènes. Le changement « total » annoncé par F. Lecomte au début du spectacle concerne l'ordre des scènes et leur remaniement. Par exemple, la répétition d'une scène ou l'extraction d'un personnageclé d'une scène et son export dans les autres, tels le monstre blanc, le monstre noir et le personnage allégorique de l'argent (« miye njo Pesa »). Les réactions du public étaient uniquement en swahili mais, comme je notais à la volée leurs interventions toujours vives, je me suis rendue compte en les retranscrivant que je le faisais plus rapidement en les traduisant directement en français qu'en les notant en swahili.

Crédits : Maëline Le Lay. 

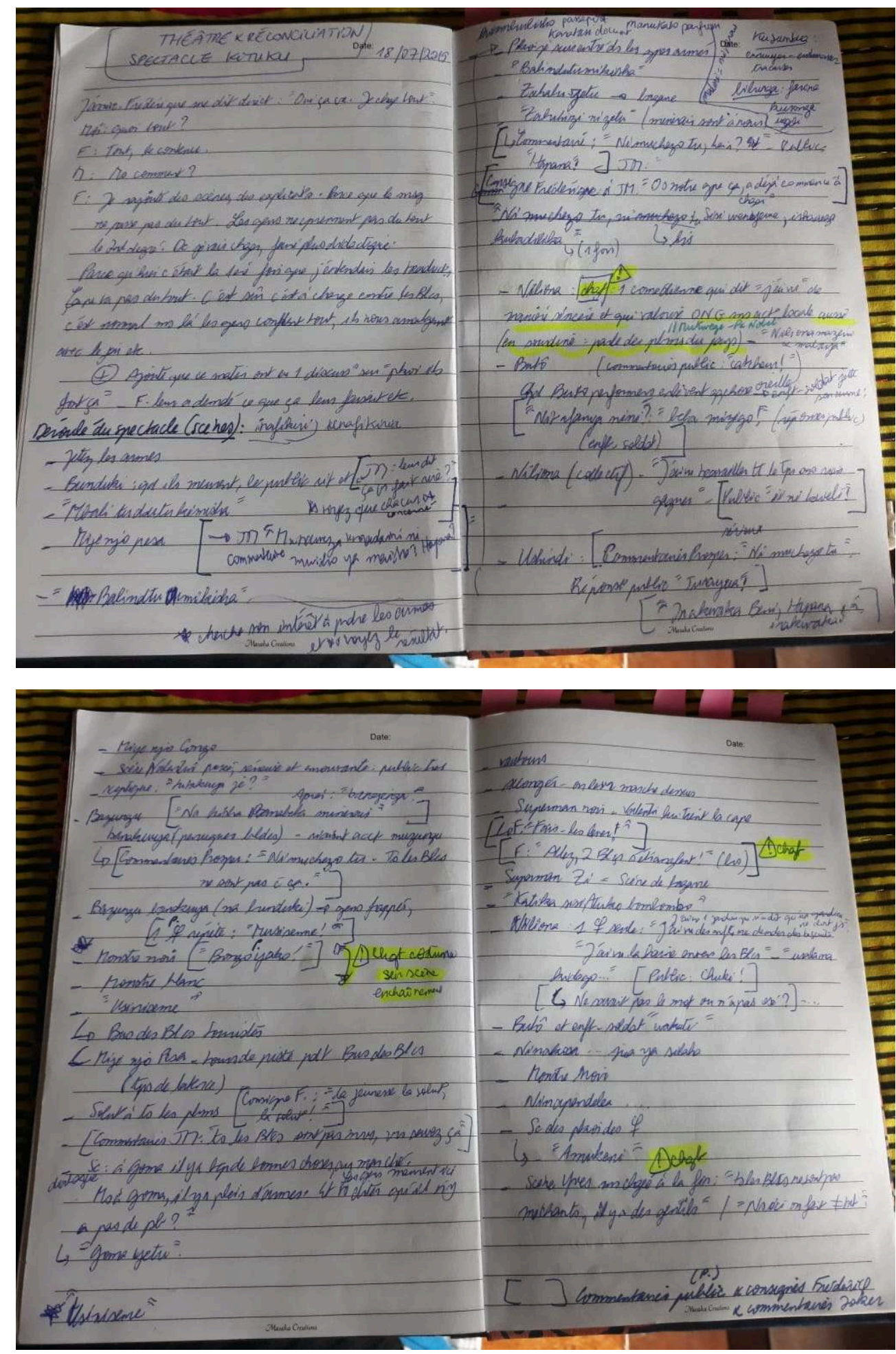


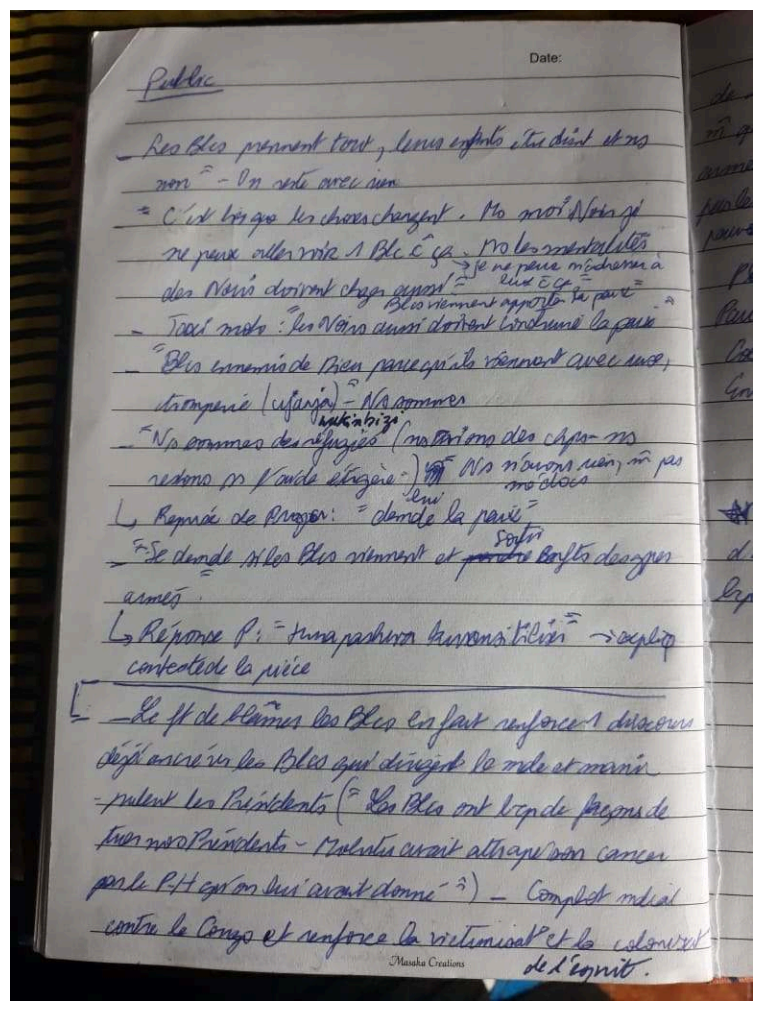

\section{NOTES}

1. Ce texte doit beaucoup aux conversations et échanges très riches que j'ai eus - et continue d'avoir - avec Aurore Vermylen et Aurore Desgranges. Qu'elles en soient ici remerciées.

2. Le texte s'ouvre sur ces mots : "Mon ami et collègue Janós Riesz m'a un jour expliqué que nous étions des Lumpensammler. Janós est un romaniste, comparatiste et philologue, et il s'occupe de l'Afrique. Le terme de Lumpensammler, emprunté à Walter Benjamin, signifie chiffonnier. Nous faisons notre miel des vieux papiers, de ce que nous ramassons, voire collectionnons... » : Alain Ricard, "Vertus de l'indiscipline : langues, textes et traductions ", discours d'ouverture des $4^{\text {es }}$ Rencontres des Études Africaines en France (REAF), 5 juillet 2016, Études Littéraires Africaines, $\mathrm{n}^{\circ}$ 42, 2016, p. 107-124. La figure du chiffonnier a pareillement inspiré Antoine Compagnon dont la conception de la littérature rejoint en partie celle d'Alain Ricard: Antoine Compagnon, Les Chiffonniers de Paris, Paris, Gallimard, coll. «Bibliothèque illustrée des Histoires», 2017. Je remercie Nicolas Martin-Granel d'avoir attiré mon attention sur ce livre.

3. Le programme de recherche soutenu par l'ANR, «Les dimensions de l'objet swahili: Textes et terrains »(2008-2011), co-dirigé par Alain Ricard et François Bart, géographe de l'Université Bordeaux-Montaigne, fut l'occasion pour Alain Ricard de mettre sa méthode à l'épreuve en dialogue avec un collectif de chercheurs en sciences humaines (essentiellement des littéraires et des géographes). Pour une présentation de ce programme de recherche et des ouvrages auxquels il a donné lieu, voir la chronique bibliographique de Marie-Aude Fouéré, «Engagez-vous, rengagez-vous dans les études sur le swahili aujourd'hui ! », Cahiers d'Études africaines, LV (3), 219, 2015, p. 587-601.

4. Il développe ici une réflexion déjà entamée dans son précédent ouvrage, Marcher avec les dragons, Paris, Zones sensibles, 2013 et Paris, Seuil, coll. « Points », 2018.

5. Tim Ingold, Faire anthropologie, archéologie, art et architecture [2013]. Traduit de l'anglais par Hervé Gosselin et Hicham-Stéphane Afeissa, Bellevaux, éditions Dehors, 2018, p. 22-23. 
6. Tim Ingold, op. cit., p. 25.

7. Karin Barber, Africa's Hidden Histories. Everyday Literacy and Making the Mind, Indianapolis, Indiana University Press, 2006.

8. Tim Ingold, op. cit., p. 32.

9. James Clifford et George Marcus, eds, Writing Culture: the Poetics and Politics of Ethnography, University of California Press, 1986; James Clifford, The Predicament of Culture: TwentiethCentury Ethnography, Literature, and Art, Harvard University Press, 1988 ; Geertz, Clifford, Ici et là-bas. L'anthropologue comme auteur [1988], Paris, Métailié, 1992. Pour un bon résumé de ce tournant épistémologique important en anthropologie, voir Michel Agier, «Le tournant contemporain de l'anthropologie », Socio, 2013, 1, « Penser global», p. 77-93: « En définitive, ce que rend possible le tournant contemporain de l'anthropologie c'est, pour l'énoncer en une formule synthétique, un savoir anthropologique plus proche d'une réflexivité de la situation ethnographique dans laquelle l'observateur est lui-même impliqué. Il s'agit de rendre ce savoir plus empathique avec les raisons des sujets en présence dans la situation et dans le contexte partagés par l'observateur. Davantage orienté, donc, par la volonté de comprendre et de décrire le pourquoi et le comment de leurs actions ici et maintenant, et ainsi de les rendre plus proches, que par celle de produire le tableau et l'herméneutique de la culture des autres, qui seraient, par cet artifice, à nouveau rendus irrémédiablement différents et lointains. Implication, réflexivité, empathie méthodologique, compréhension, décentrement sont les mots-clés de la révision de l'anthropologie contemporaine. »

10. Voir Didier Fassin, "The ethical turn in Anthropology. Promises and uncertainties ", HAU. Journal of Ethnographic Theory, 4 (1), 2014, p. 429-435.

11. Aurore Vermylen, «Par-delà les émotions et la raison. Les apports de l'auto-analyse comme méthode de compréhension de nos terrains ", revue e-Migrinter, [En ligne], 18 | 2019, mis en ligne le 11 septembre 2019, consulté le 30 septembre 2019. URL: http://journals.openedition.org/emigrinter/1781 ; Pierre-Joseph Laurent, Devenir anthropologue dans le monde d'aujourd'hui, Paris, Karthala, 2019 ; Jean-Baptiste Lanne, Des vies en veille. Géographies abandonnées des acteurs quotidiens de la sécurité à Nairobi. Thèse de doctorat en géographie soutenue à l'Université de BordeauxMontaigne, sous la co-direction de Bernard Calas et Myriam Houssay-Holzschuch, 28 novembre 2018.

12. Voir par exemple l'expérience de Bernard Müller, se rendant au Nigeria, espérant y trouver les formes de théâtre sur lesquelles il s'était documenté depuis la France. Son livre, La tradition mise en jeu, fait le récit - éminemment réflexif - de cette rencontre déceptive avec son terrain de recherche: Bernard Müller, La Tradition mise en jeu. Une anthropologie du théâtre yoruba, La Courneuve, éditions Aux lieux d'être, coll. « Mondes contemporains », 2006.

13. À propos de l'histoire de la constitution du «classique africain » voir Claire Ducournau, La Fabrique des classiques africains. Écrivains d'Afrique subsaharienne francophone (1960-2012), Paris, CNRS éditions, 2017.

14. Alain Ricard, Texte moyen, texte vulgaire. Essai sur l'écriture en situation de diglossie. L'écrivain public Félix Couchoro et les comédiens ambulants du Happy Star Concert. Lomé, Togo. 1962-1977, Bordeaux, Thèse de doctorat, Bordeaux III. Sous la direction de Robert Escarpit. 1981 ; Alain Ricard, Karin Barber, et John Collins, West African Popular Theatre, Bloomington/ Indianapolis, James Currey/Indiana University Press, 1997.

15. Alain Ricard, Le Principe d'Asihu, 51', CNRS images [1982], 2011. Voir aussi Bankole Bello, Wole Soyinka, poète-citoyen, avec Alain Ricard, Arte/FR3/La Sept, conseiller littéraire, 1993.

16. Johannes Fabian, Power and Performance. Ethnographic explorations, Proverbial Wisdom and Theater in Shaba, Zaïre, Madison, The University of Wisconsin Press, 1990 ; Karin Barber, The Generation of Plays. Yoruba Popular Life in Theatre, Indianapolis, Indiana University Press, 2000.

17. Céline Gahungu, "Le Concours théâtral interafricain : quelles archives? Quels usages?», article dans ce numéro. 
18. Brian Valente-Quinn, et Ferdinand De Jong, «Ruines d'utopie, l'école William Ponty et l'Université du futur africain ", Politique africaine, n 135, octobre 2014, p. 71-93 ; Tobias Warner, "Para-literary Ethnography and Colonial Self-Writing ", Research in African Literatures, vol. 47, issue 1, printemps 2016. Pour un aperçu de l'orientation vers la pratique et la création théâtrale de l'école normale William Ponty, voir: "Le théâtre indigène et la culture franco-africaine », numéro spécial de l'Éducation Africaine (Bulletin de l'enseignement de l'AOF), Dakar, 1937.

19. Voir à ce sujet les chapitres 1 et 2 d'Alain Ricard, Littératures d'Afrique noire. Des langues aux livres, Paris, CNRS éditions/Karthala, 1995.

20. Il est important de noter que cet état des lieux, assez sombre, ne prévaut plus aujourd'hui. En outre, certains artistes ont déjà dépassé cette barrière et s'accommodent des lieux existants. Yves Ndagano, comédien, metteur en scène et musicien a ainsi lancé le Festival du théâtre du Kivu (qui en est cette année à la $3^{\mathrm{e}}$ édition) qui se déroulait l'année dernière dans l'enceinte de l'ex-Alliance française, aujourd'hui rebaptisée "Espace culturel francophone », un lieu doté d'une des seules salles de la ville pourvues d'une scène rudimentaire. Les spectacles avaient lieu à l'intérieur comme à l'extérieur dans la parcelle où une scène - sonorisée et éclairée par des projecteurs avait été montée sous les arbres, «à la manière des Recréâtrales de Ouagadougou », clamait Yves qui y avait participé en 2014 et dont l'exploitation théâtrale du plein air l'a fortement inspiré.

21. « Writing is not what confers textuality » ( Ce n'est pas l'écriture qui confère la textualité ») : Karin Barber, The Anthropology of Texts, Persons and Publics. Oral and written culture in Africa and Beyond, Cambrige, Cambridge University Press, 2007, p. 1.

22. "Les gens qui ont une formation en littérature européenne peuvent être tentés d'affirmer (et l'ont souvent fait) que chaque culture a une catégorie qui correspond à la "littérature", que chaque "littérature" est caractérisée par l'unité, la dimension fictionnelle, le langage poétique ou le travail sur l'imagination; et que le fait de répondre à tous ces critères est le moyen de comprendre le texte. Mais un des buts de l'anthropologie des textes est d'élargir l'éventail de relations que les textes peuvent établir entre l'écrivain/l'orateur et le lecteur/auditeur/ spectateur, et les voies par lesquelles ils peuvent être chargés de sens. Une des assertions centrales de ce livre place la textualité au cœur de l'analyse : si un texte verbal a quelque chose à nous dire à propos d'une société, d'une expérience sociale ou de valeurs culturelles, cela ne peut que passer que par sa textualité, sa manière spécifique d'être un texte, on ne saurait faire l'économie de cette dimension " : Ibidem, p. 13.

23. Une performance ne peut qu'être performance de quelque chose, en l'occurrence d'un texte, que celui-ci soit écrit ou oral : "This is to render discourse object-like: by making it the focus of commentary and exegetical attention, or by presenting text as quotable, thus foregrounding the perception that these words pre-existed their present moment of utterance and could also continue to exist after it. [...] I want to emphasize that in constituting text as object-like, these African genres do not forego the fluidity and improvisatory quality that performance theory has so successfully explored. Rather, it is the very consolidation of chunks of examinable, quotable, repeatable text that makes possible the dynamic processes of fluid incorporation, re-inflection, and recycling" ( Il s'agit de faire du discours un objet: en en faisant le point focal du commentaire et de l'exégèse, or ou présentant le texte comme citable, privilégiant ainsi la perception que ces mots pré-existent au moment présent de leur émission et peuvent aussi continuer à exister au-delà. Je voudrais mettre l'accent sur le fait qu'en faisant des textes des objets, ces genres africains ne renoncent pas à la fluidité et à la capacité d'improvisation que la théorie de la performance a explorée avec succès. C'est plutôt la consolidation de morceaux de textes citables et faits pour être répétés qui rend possible les processus dynamiques d'incorporation, de ré-inflexions et de recyclage. ») : Ibidem, p. 268.

24. «Concevoir le style comme modalité, variation modale de la vie sur elle-même, oriente vers ce genre particulier d'attention et de description qui consiste à reconnaître dans tout être l'engagement d'un mode d'être, dans tout faire l'engagement d'une manière de faire. Cela conduit 
à considérer la vie elle-même comme une dynamique d'institution d'une multiplicité de formes, ou plutôt de modes, de tons [...] : le réel y est fondamentalement compris comme une foule de modes d'être. [...] Tout l'enjeu ici consiste à [...] regarder ces petites expressions (modes de, façons de, manières de...) comme [...] une force de possibilisation inhérente à la vie-même. [...] Ce partage modal du sensible est l'ouverture, toutes portes battantes, de l'attention stylistique à la vie ellemême » : Marielle Macé, Styles. Critique de nos formes de vie, Paris, Gallimard, coll. " nrf essais ", 2016, p. 39-40.

25. Gay McAuley, «Not Magic but Work. Rehearsal and the Production of Meaning ", Theatre Research International, vol. 33, n 3, 2008, p. 276-288, p. 286. Gay McAuley se réfère à cet extrait des écrits d'Antoine Vitez qu'elle cite (p. 286) : «Il y a un champ imaginaire du théâtre, le temps des répétitions est une sorte de cloche à plongeurs, comme une bulle à l'intérieur de notre vie, d'une certaine manière une drogue. Le temps des répétitions est très précieux pour les acteurs, le metteur en scène, toute l'équipe de travail : à ce moment-là se crée toute une vie de métaphores, de comparaisons, d'idées lancées qui parfois avortent et parfois rebondissent. À la différence d'une mauvaise, une bonne mise en scène est une mise en scène qui travaille, qui bêche ce champ imaginaire, qui ne laisse pas avorter ou dépérir les idées qui naissent au temps des répétitions, l'imprévu, la fraîcheur même de l'invention des acteurs. (Antoine Vitez, « Antoine Vitez », Cahiers Théâtre Louvain, 46 [octobre 1981], p. 96).

26. Ibidem. «Percevoir ainsi la répétition comme un lieu de relations interpersonnelles complexes, un lieu de travail, un creuset facilitant la créativité collective, exige une approche très différente de l'effort essentiellement herméneutique décrit dans le paragraphe précédent. La recherche théâtrale traditionnelle fournit peu de conseils méthodologiques pour faire face à une telle tâche, mais l'ethnographie a été une riche source de concepts analytiques, de procédures et d'idées. J'ai repris le concept de "description épaisse" de Clifford Geertz, et c'est ce qui résume le mieux ce que l'étude des répétitions doit aborder selon moi».

27. "Quelles sont les étapes d'un texte avant sa publication? Quand et comment l'auteur hésitet-il, fait-il des erreurs, fait-il des détours et prend-il des décisions? En se concentrant sur l'écriture "en cours" et sur le travail créatif derrière la littérature, l'analyse génétique propose d'analyser le processus d'écriture lui-même, en utilisant les traces qui restent sous forme de manuscrits ou de brouillons, par exemple » : Josette Féral, «Introduction: Toward a genetic study of performance - Take $2 »$, Theatre Research International, vol. 33, n 3, 2008, p. 223-233, p. 225.

28. Augusto Boal, Théâtre de l'opprimé [1970], Paris, La Découverte, 2003. Traduit du brésilien par Dominique Lémann.

29. Sur l'histoire du théâtre pour le développement, voir par exemple : Kees Epskamp, Theatre for Development. An Introduction to Context, Applications and Training, London \& New York, Zedbooks, 2006 ; Penina Mlama Muhando, Culture \& Development. The Popular Theatre Approach in Africa, Uppsala, The Scandinavian Institute of African Studies, 1991 ; ainsi que la partie consacrée au théâtre dans Ngugi wa Thiong'o, Decolonising the Mind. The Politics of Language in African Literature, Oxford, James Currey, 1986. Pour ce qui est de recherches récentes, citons : Aurore Desgranges, "Malaise dans la sensibilisation : autorité didactique et performance au Burkina Faso », Colloque "Pratiques artistiques contemporaines d'Afrique: formes et enjeux politiques", Bordeaux, Musée d'Aquitaine, vendredi 18 mai 2018.

30. Association sans but lucratif.

31. Parmi les principaux commanditaires de Frédérique Lecomte, citons les ONG suivantes : RCNJustice et démocratie (ONG belge), Search for Common Ground (ONG américaine), La Benevolencija Grands Lacs (ONG basée aux Pays-Bas). Pour en savoir plus sur le nœud de conflits interdépendants en Afrique des Grands Lacs, voir Filip Reytjens, The Great African War. Congo and Regional Geopolitics (1996-2006), Cambridge, Cambridge University Press, 2009 et Gérard Prunier, Africa's World War. The Rwandan Genocide and the Making of a Continental Catastroph, Oxford, Oxford University Press, 2009. Pour une lecture ciblée de l'Est du Congo comme épicentre de la « grande 
guerre mondiale africaine », voir l'exposé remarquablement synthétique de Séverine Autesserre, The Trouble with the Congo. Local Violence and the Failure of International Peacebuilding, Cambridge, Cambridge University Press, Cambridge Studies in International Relations, 2010, p. 47-51.

32. Frédérique Lecomte (dir.), Théâtre \& Réconciliation. Méthode pour une pratique théâtrale dans une zone de conflit, Bruxelles, La Lettre volée, coll. « Essais », 2016.

33. «Un texte est créé quand les instances de discours, en étant rendues détachables de leur contexte immédiat d'émission, sont rendues disponibles pour la répétition ou la re-création dans d'autres contextes. En d'autres termes, il y a des bouts/segments de discours qui peuvent être reproduits et ainsi transmis au-delà du temps et de l'espace. »: Barber, op. cit., p. 22. Et, p. 2 : " text in the sense in which I am using it in this book, is utterance (oral or written) that is woven together to order to attract attention and to outlast the moment.» («le texte, au sens où je l'entends dans ce livre, est l'énoncé (oral ou écrit) qui est tissé pour retenir l'attention et perdurer au-delà du moment »).

34. Sur le rôle particulier du chœur dans les spectacles de Théâtre \& Réconciliation/ Frédérique Lecomte, je me permets de renvoyer à mon chapitre : Maëline Le Lay, « Humanitarian Theatre in the Great Lakes Region: In Pursuit of Performativity », dans Chérie Rivers Ndaliko \& Samuel Mark Anderson (éd.), The Art of Emergency. Aesthetics and Aid in African Crises, Oxford \& New York, Oxford University Press, à paraître en décembre 2019.

35. À l'exception notable de Sénouvo Agbota Zinsou, rebaptisé Nestor Zinsou, son étudiant qui devint son ami et proche collaborateur. Il l'accompagna dans ses enquêtes de terrain auprès des troupes de concert-party et participa aux opérations de transcription et traductions des pièces. Plus tard, Alain Ricard lui consacra un film (« Nestor Zinsou à Bayreuth », HDV, 24 min., 2013) et un livre, Wole Soyinka et Nestor Zinsou. De la scène à l'espace public. Religion et politique, Paris, Karthala, coll. « Lettres du sud», 2015.

36. Voir Alain Ricard, Félix Couchoro. Naissance du roman africain (1900-1968), Paris, Présence africaine, 1987.

37. Je lui ai d'ailleurs sans doute emprunté ce point aveugle d'une analyse du terrain qui aurait gagné à être plus réflexive, en ne justifiant pas davantage, dans ma thèse de doctorat comme dans le livre que j'en ai tiré, mon propre choix de considérer dans le même corpus des textes écrits (et la plupart du temps publiés) en français et des textes semi-oraux en swahili.

38. Johannes Fabian, op. cit., p. 89.

39. Id., p. 88. «Il s'agit avant tout [...] de protocoles faits d'échanges communicatifs (surtout verbaux) réels, ainsi que de "performances", allant de la narration à des récitations poétiques et mythiques, en passant par des rituels, des enregistrements de transactions et des litiges. D'autres types peuvent bien sûr être ajoutés à cette liste, comme les récits autobiographiques, les descriptions et instructions relatives au travail et à la production, ainsi que les paroles des chansons. »

40. Id., p. 91. «Il serait arbitraire d'isoler un événement du processus auquel il appartient et de présenter la représentation filmée comme la pièce définitive. Pour cette seule raison, il n'est pas possible de déclarer qu'un texte est définitif ».

41. "En d'autres termes, lorsque l'ethnographie est dite "texto-centrée", cela concerne avant tout son fondement épistémologique ; cela ne signifie pas que la collecte de textes soit le but principal de la recherche. Un texte, pour devenir "collectable", doit être pertinent pour un projet qui est toujours plus large que ce qu'un texte spécifique peut documenter. Ceci s'applique également au document choisi pour l'interprétation dans ce livre. [...] Peut-être pas au moment où elle $\mathrm{a}$ eu lieu, mais certainement après, la conversation avec Kahenga a fait partie d'un corpus de textes, un terme qui peut avoir un sens technique en théorie littéraire mais qui ne perd jamais complètement ses connotations métaphoriques. Comme un corps, un corpus a une taille, un volume, un poids, l'articulation des parties et des membres; tant qu'il est vivant, il grandit et change. Ces derniers, la croissance et le changement, correspondaient certainement au corpus de 
documents que j'ai produits (plus souvent que je ne l'ai trouvé) dans le cadre de mon travail d'ethnographe »: Johannes Fabian, Ethnography as Commentary. Writing from the Virtual Archive, Durham \& London, Duke University Press, 2008, p. 14-15. En passant, on comprendra que Johannes Fabian défend une conception de l'ethnographie à la portée bien plus large que celle que lui prête Tim Ingold.

42. Id., p. 40. " Il y a une autre raison de mettre l'accent sur le "making". Cela exprime l'idée que la production de connaissances implique l'objectivation. Seuls les sujets peuvent savoir mais pour être présentées et partagées, les expériences et les idées subjectives doivent être objectivées. Elles doivent exister matériellement, s'incarner dans le sujet connaissant, dans les médiations sensibles qui permettent aux sujets de communiquer par le langage, et dans toutes les autres choses et pratiques que nous appelons habituellement culture. Les textes font partie des objets nécessaires à la production de la connaissance ethnographique ».

43. Nathalie Heinich, L'Élite artiste. Excellence et singularité en régime démocratique, Paris, Gallimard, 2005.

44. Alain Ricard, Wole Soyinka et Nestor Zinsou. De la scène à l'espace public. Politique et religion, p. 7.

45. À noter que la dimension collective du travail de terrain, rendue possible par une collaboration avec des " assistants de recherche ", n'est pas toujours pleinement assumée par les chercheurs du Nord qui ont fréquemment recours (dans certaines disciplines plus que dans d'autres) à des assistants locaux pour mener leurs recherches dans le Sud, un travail hélas souvent minoré à de nombreux égards. Citons en guise d'exemple ce collectif belgo-congolais de chercheurs qui travaille actuellement à dénoncer l'invisibilisation des chercheurs du Sud dans le dispositif de productions du savoir académique en sciences sociales. Voir la «Bukavu series » du GIC (Governance and Conflict Network) de l'Université de Louvain et de l'ISDR (Institut Supérieur de développement rural) de Bukavu, et notamment leur introduction, «Invisible Voices in the Production of Knowledge. Introduction to the (Silent) Voices Bukavu Series» (juin 2019) : https://www.gicnetwork.be/introducing-the-bukavu-series-invisible-voices-in-the-productionof-knowledge/

46. En sus de cette traduction collective, Marcel Kalunga, professeur de kiswahili et de linguistique bantoue à l'UNILU (Université de Lubumbashi), traducteur chevronné du swahili au français et vice versa, avait relu ces traductions et m'avait aidée à éclaircir l'un ou l'autre point demeuré obscur.

47. " "Formes de vie", "manière de vivre", "manière d'être", "styles de vie" sont des formules déterminantes de la philosophie moderne, de Wittgenstein à Stanley Cavell [...] Dans la plupart des cas, ces notions [...] renvoient fondamentalement à un intérêt pour ce qu'Agamben a appelé la vie qualifiée, qui prend sens dans son opposition première à une vie nue (le seul fait d'être en vie). La vie qualifiée, c'est la forme de vie, la manière de vivre, la vie "telle ou telle" ; une vie dont le sens est immanent au "comment", une vie dont le sens est tout entier contenu dans son mode, une vie dont la forme est puissance " : Marielle Macé, op. cit., p. 49.

48. «Cet objet, fondamentalement sociologique et donc historique, c'est la manière dont les sociétés se connaissent en se parlant et en s'écrivant, dont l'homme-en-société se narre et s'argumente. Cet objet, c'est une science du discours global. [...]. Autrement dit, l'effet 'littérature' ne peut être jugé et mesuré que par rapport au système socio-discursif global dans lequel il s'engendre»: Marc Angenot, "Que peut la littérature ? Sociocritique littéraire et critique du discours social », dans Jacques Neefs \& Marie-Claire Ropars (éd.), La Politique du texte. Enjeux sociocritiques. Pour Claude Duchet, Lille, Presses universitaires de Lille, 1992, p. 14.

49. Marielle Macé, op. cit., p. 48. À cette conception de la littérature comme lieu privilégié de l'observation des formes du vivre, elle ajoute, p. 49, « qu'il y a bien-sûr de la littérature dans les sciences humaines, lorsque précisément elles font de l'écriture un instrument de vérité, lorsqu'avec l'écriture elles ne se veulent pas plus élégantes ou plus subjectives, mais plus justes, et plus conscientes de la pluralité des sens qui s'instituent dans les pratiques elles-mêmes ». 
50. François Laplantine, Penser le sensible, Paris, Pocket, coll. « Agora » 2018, p. 29.

51. Tim Ingold, op. cit., p. 53.

52. Ibidem, p. 54

53. Ibidem, p. 54-55.

54. Voir aussi : Didier Nativel, «Les sens de la nuit. Enquête sur les sensorialités urbaines coloniales à Madagascar et au Mozambique", Sociétés politiques comparées. Revue européenne d'analyse des sociétés politiques [En ligne], dossier « Nuits urbaines ", $\mathrm{n}^{\circ} 38$, jan-avril 2016. Il y étudie les sensorialités nocturnes coloniales en s'appuyant sur les écrits de deux poètes, Jean-Joseph Rabearivelo et José Craveirinha

55. François Laplantine, op. cit., p. 58.

56. Didier Nativel, chap. 8, "La ville comme déploiement du sensible ", Contribution à une histoire des sociétés et des espaces urbains de l'océan Indienoccidental (xixe-xxe), HDR d'histoire, Université Paris-Diderot Paris 7, 2013.

57. François Laplantine, op. cit., p. 59.

58. Didier Nativel, op. cit,

59. Kathleen Stewart, "Granite", Society for Cultural Anthropology, for the series "Correspondance", 18 février 2019 : https://culanth.org/fieldsights/granite. Pour un aperçu plus large de son travail et sa méthode, voir son livre : Kathleen Stewart, Ordinary Affects, Durham \& London, Duke University Press, 2007.

60. François Laplantine, op. cit., p. 58.

61. Anna Tsing part de son expérience olfactive et gustative des matsutakes (voir notamment "Interlude: humer», p. 87-97) pour développer une pensée critique du capitalisme et de l'écologie, dans le sillage de celle élaborée dans son essai le plus connu, Frictions. Anna Tsing, Le Champignon de la fin du monde. Sur la possibilité de vivre dans les ruines du capitalisme, préface d'Isabelle Stengers, traduit par Philippe Pignarre, Paris, La Découverte, 2017.

62. «Ce petit "quelque chose en plus" qui fait que nous sommes traversés par nos réalités de terrain, que nous sommes "construits" par nos réalités de terrain. Encore faut-il être capable de repérer et de faire la part des choses entre les émotions qui nous concernent et celles qui concernent nos terrains. J'essaye donc, de faire régulièrement le point et de comprendre "ce qui m'appartient" et "ce qui appartient à ce que je vais décrire" - démarche qui probablement s'estompera au fil de mes années de terrain, puisque la distinction entre les deux sera de plus en plus mince »: Aurore Vermylen, "Par-delà les émotions et la raison. Les apports de l'autoanalyse comme méthode de compréhension de nos terrains », p. 13. Elle analyse dans son article les motifs de l'irruption d'émotions étrangères à son fonctionnement habituel qui l'ont « submergée » sur son terrain : une colère intense au Nord-Kivu et un sentiment de paranoïa au Burundi.

63. Olivier Marcel, «Le Nairobi des poètes: une cartographie des pratiques littéraires de la génération Kwani ? ", Études Littéraires Africaines, n 31, 2011.

64. La question des contours de l'Afrique des Grands Lacs a fait l'objet de discussions importantes dans le champ académique. Jean-Pierre Chrétien qui a dressé l'histoire de sa construction politique (Jean-Pierre Chrétien, L'Invention de l'Afrique des Grands Lacs. Une histoire $d u X^{e}$ siècle, Paris, Karthala, 2010), traite sous cette étiquette, dans un autre de ses essais devenu un classique de l'histoire de la région, L'Afrique des Grands Lacs. Deux mille ans d'histoire (Paris, Aubier, 2000), du Rwanda et du Burundi dont la plupart des chercheurs s'accordent à faire le cœur de cette région des Grands Lacs, mais aussi davantage de l'Ouganda que de l'Est de la RDC. M'intéressant à la construction institutionnelle et politique de ce terme qui tend, de manière croissante, à envisager le Rwanda, le Burundi et l'Est de la RDC comme des parties constituantes d'une même région, je m'en tiens pour ma part à l'étude de ces trois pays.

65. Maëline Le Lay, "Géocritique de la RDC», dans Bi Kacou Parfait Diandue (dir.), «Une géocritique de l'Afrique. Mutations et stabilité de la spatialité et de la temporalité dans le locus 
africain ", Revue Baobab (Université Cocody d'Abidjan), 2009. L'analyse du Fleuve Congo comme topos littéraire a pu être développée collectivement au cours de la journée d'étude co-organisée avec Jean-Pierre Orban au Musée du Quai Branly le 2019: Maëline Le Lay \& Jean-Pierre Orban (dir.), «Écrire le Fleuve Congo après Conrad », Continents manuscrits, $n^{\circ}$ 11, 2018.

66. Johannes Fabian, Power and Performance, p. 4. Il reprend un extrait de son journal de bord au début du processus : "Whatever happened [on that afternoon], here is a new ethnography" - the ethnographers's interpretative idea [...] is taken up, collectively discussed, cast into a play, tested on a public etc.» / "Quoi qu'il se soit passé d'autre [cet après-midi-là], nous avons là une "nouvelle ethnographie"- la problématique posée par l'ethnographe est reprise, collectivement discutée, intégrée à une pièce de théâtre, testée sur un public ».

67. Jean-Baptiste Lanne, op. cit., p. 218.

68. Ibidem, p. 233.

69. Ibidem, p. 236.

70. «Les askaris [«soldats» en swahili; au Kenya c'est ainsi qu'on désigne les gardes des résidences], quant à eux, ne décident pas de nos entrevues : qu'ils souhaitent que je passe ou que je ne passe pas, ils doivent s'en remettre à l'incertitude de ma venue. Lors de la phrase de création poétique au contraire, le simple fait d'être liés à un travail commun - l'avancement du poème, qui exige des entrevues fixes - comporte une dimension d'engagement permettant de rétablir, même sous forme de fiction, une forme d'équilibre » : Ibidem, p. 240.

71. Almuth Grésillon, "De l'écriture du texte de théâtre à la mise en scène", Cahiers de praxématique, $\mathrm{n}^{\circ} 26$, «Les mots et la scène », 1996, document 4, mis en ligne le $1^{\mathrm{er}}$ janvier 2015, consulté le 4 décembre 2018. URL : http://journals.openedition.org/praxematique/2979

72. Ibidem.

73. Au sujet du second rôle assigné à l'anthropologue, voir Aurore Vermylen, «Par-delà les émotions et la raison. Les apports de l'auto-analyse comme méthode de compréhension de nos terrains ", e-migrinter, $\mathrm{n}^{\circ} 18$, "L'ethnographie en migration(s)», 2019, consulté le 20 octobre 2018. URL : https://journals.openedition.org/e-migrinter/1781.

74. « Dans les ethnographies qui prétendent livrer des textes ou des données nus, la nudité n'est qu'un autre costume. En tant qu'écrivain, l'anthropologue façonne et forme son matériau. Il se distingue de l'auteur de fiction, non pas par ce qu'il présente, mais par ce dont il a besoin pour justifier ses présentations en tant que contributions à un ensemble de langages »: Fabian, Power and Performance, p. 87.

75. " The ethnograph is no longer that of a questioner; he or she is but a provider of occasions, a catalyst in the weakest sense and a producer (in analogy to a theatrical producer) in the strongest. Victor Turner, pursuing a similar line of thought, has called the ethnographer an "ethnodramaturg" : Ibidem, p. 7.

76. Pour un aperçu de la méthode de Turner, voir la présentation de sa pédagogie dans son article, co-écrit avec Edith Turner, «Performing Ethnography». Les époux y relatent leurs expériences pédagogiques de performance d'un mariage à l'américaine avec les étudiants de Virginia University ainsi qu'un rite observé chez les Ndembu de Zambie, les deux expériences leur ayant permis de comprendre par la performance ce qu'ils n'avaient pas compris (ou ce dont ils n'avaient pas conscience) sur le terrain (ou dans la vie ordinaire, s'agissant du mariage) : Victor Turner et Edith Turner, « Performing Ethnography », The Drama Review: TDR, vol. 26, n², «Intercultural Performance », 1982, p. 33-50.

77. Bernard Müller, «Le terrain : un théâtre anthropologique », dans Christian Biet et Sylvie Roques (dir.), Communications, « Performance. Le corps exposé », n 92, 2013, p. 75-83. 


\section{RÉSUMÉS}

Prenant appui sur une expérience d'observation participante de longue durée en Afrique des Grands Lacs dans le cadre d'une recherche sur les formes littéraires et théâtrales, comme sur des travaux analogues de chercheurs qui y font écho, cet article interroge le statut des textes étudiés, leur textualité particulière en tant que matière essentiellement orale et partiellement improvisée. Il réfléchit aussi à la manière dont l'implication physique du chercheur sur son terrain, ses interactions et les effets qu'elles peuvent avoir sur le déroulement du processus théâtral, doivent être pris en compte dans l'analyse des spectacles étudiés.

Drawing on a longstanding participant observation in Africa's Great Lakes researching on literary and theatrical forms, as well as on similar works by researchers, this article questions the status of texts, their particular textuality as being essentially oral and partially improvised. It also reflects on how the researcher's physical involvement in his or her field, his or her interactions and the effects they can have on the development of the theatrical process, must be taken into account in the analysis of the performances.

\section{INDEX}

Mots-clés : théâtre, Afrique, terrain, textualité, sensible

Keywords : theatre, Africa, fieldwork, textuality, sensory studies

Index géographique : Afrique

\section{AUTEUR}

\section{MAËLINE LE LAY}

Chargée de recherche CNRS à l'IFRA-Nairobi. 